\title{
Stock Price Reactions to Earnings Announcements in Indian Stock Market
}



DOI: $10.26573 / 2017.11 .3 .1$

Volume 11, Number 3

September 2017, pp. 151-178

\author{
Janet Jyothi Dsouza \\ Ballari Institute of Technology and \\ Management \\ (janetjyothidsouza@gmail.com)
}

T. Mallikarjunappa

Mangalore University

(tmmallik@yahoo.com)

Efficient Market Hypothesis theory (EMH) which states that an investor cannot consistently beat the market based on any set of information whether it is historical, publically available information or private information is examined. The investors' expectation on the extent of excess return that they would make from trading in the stock market is based on several factors and one of them is quarterly earnings announcement news. It was examined that whether there is any significant relationship between stock returns and quarterly earnings announcement. For this purpose, we employ event study methodology. The mean adjusted model, market adjusted model and market model are used to measure the abnormal performance. t test is used (Brown \& Warner, 1985) for statistical significance and Runs and Sign test for testing the hypotheses. Cohen et al. (1983) methodology is also used to see the price adjustment process during the quarterly earnings announcement. The results show that Cumulative Average Abnormal Returns (CAARs) are statistically significant for most of the days in the event window. The findings of the study support the prediction that quarterly earnings information contains information value which is not reflected in security prices and therefore, traders can outperform the market based on quarterly earnings announcements in Indian stock market.

Keywords: Efficient Market Hypothesis theory (EMH), Excess Return, Indian Stock Market, Cumulative Average Abnormal Returns (CAARs), Quarterly Earnings Announcements

\section{Introduction}

The concept of stock market efficiency has been investigated since 1950s and it has been regarded as one of the important areas of research in modern finance. The development of Efficient Market Hypothesis (EMH) (Fama 1965, 1970) has created the interest among the researchers to examine its validity. Many researchers investigated the stock market reaction to informational disclosure by considering various corporate announcements such as stock split, mergers and acquisition, dividend announcement etc. Stock price response to earnings announcements has received considerable attention as earnings are considered as the firms' performance indicator. EMH states that an investor cannot beat the market based on any set of new information, whether it is historical, publically available information or private 
information. The investors' expectation on the extent of excess return that they would make from trading in the stock market is based on several factors and one of them is quarterly earnings announcement news. It has been observed that, during the earnings announcement, stock prices usually rise and increase price volatility. The earnings announcements occur in regular intervals and it provide good opportunity to test whether these announcements generate predictable returns to the investors. As the earnings contain information and influence the stock prices, the investors wait for the earnings announcement season to make money. The investors forecast the earnings on pre-announcement drift, announcement effect and on post announcement drift. There are several studies such as Ball and Brown (1968), Brown and Kennelly (1972), Woodruff and Senchack (1988), Bernard and Thomas (1989), Cornell and Landsman (1989) and Bernard and Thomas (1990) that empirically showed that earnings contain information content and traders gained trading on this information flow. The disclosure of accounting numbers of listed companies has significant influence on stock market. This study provides empirical evidence on how the stock market reacts to earning announcement in the emerging Indian stock market. We examine whether there is any significant relationship between stock returns and quarterly earnings announcements news. This paper is organized as follows: section 2 provides a review on literature, section 3 discusses the objectives and hypotheses of the study, section 4 discusses the sample and data, section 5 presents the results and analysis. Finally the conclusions are given in section 6 .

\section{Literature Review}

The stock market response to earnings announcement has gained lot of attention in modem finance literature. The previous empirical studies are reviewed in this section. Event studies have a long history and a wide range of applications. One of the first studies of this form was Dolley (1933), where the price effects of stock splits are examined. Similar studies done by Ball and Brown (1968), and Fama et al. (1969) introduced the abnormal returns model which is very popular and widely applied today. Ball and Brown (1968) was the first study to find abnormal returns of firms with positive earnings news which continued to drift upward after the earnings announcements and that the opposite is true for firms with negative news. Beaver (1968), Brown and Kennelly (1972), Foster (1977), Joy, Litzenberger, and McEnally (1977) and Nichols and Tsay (1979) examined the information content of earnings announcements, and suggested that when there is new information arrival, volume will be larger and price change will reflect the market's overall expectations regarding this information. Foster and Vickrey (1978), Woolridge (1983), Grinblatt et al. (1984), Lakonishok and Vermaelen (1986), Lonie et al. (1996) document considerable positive abnormal returns around the announcement dates of stock dividends which are consistent with the semi-strong form of market efficiency. Patell and Wolfson (1984), Jennings and Starks (1985), and Barclay and Litzenberger (1988) examine the price response to corporate announcements such as earnings, dividends, and seasoned equity offerings and found significant abnormal returns. Watts (1978), Rendleman et al (1982), Foster and Shevlin (1984), Bernard and Thomas $(1989,1990)$ found that stock prices do not adjust rapidly to the new information flow contained in the earnings announcement. These studies have discussed the market asymmetry in response to good and bad news of earnings announcement. The results showed that there is no relationship between stock 
volatility and speed of price adjustment when earnings announcements are good news and negatively related when they are bad news. Foster and Shevlin (1984) explained 'post-earnings-announcement drift' and concluded that stock prices fail to adjust abnormal returns fully for new information and have failed to resolve the anomaly. Kormendi and Lipe (1987), and Easton and Zmijewski (1989) supported the existence of efficient markets. William and Patricia (1991) argue that the earnings announcements contain some information which is not available to the public. Ball and Kothari (1991) found significant excess return which will be generated on the announcement day because earnings announcement usually include information which are not available to the public. Jegadeesh and Livnat (2006) demonstrated that price announcements contain information and are not available to the market and the stock price cannot fully reflect all the information released to the public, which is against semi-strong form EMH. Menike and Wang (2013) found positively insignificant abnormal return and cumulative abnormal return during the earnings announcement. The positive reaction towards the earnings announcement attributed the favorable information to the investors. Truong and Corrado (2014) found significant abnormal returns to earnings information announcements in US market. Sulistiawan et al. (2014) empirically examined the relationship between investors' response towards earnings announcement and technical analysis signals of Indonesia. The results showed that there is a negative relationship reaction to a technical analysis signal before an earnings announcement. Jansen and Nikiforov (2016) documented abnormal returns in the week before the earnings announcement. Further, they found abnormal returns in excess of $1.3 \%$ over a two day-window. Frank et al. (2016) investigated the effect earnings announcement on stock prices of manufacturing firms of Ghana Stock Exchange. They used event study methodology of 21 days event window and 60 days of estimation period. The results showed that there is no effect of earnings announcement on stock prices of manufacturing firms and the market is semi-strong form inefficient.

In further discussion, the studies of Efficient Market Hypothesis (EMH) which have used different corporate announcements including $\mathrm{P} / \mathrm{E}$ effect, dividend announcements, bonus issue, equity rights etc are considered. Basu (1975) argue that opportunities for earning "abnormal" returns were afforded to investors. Tax-exempt as well as tax-paying investors, who entered the securities markets with the objective of rebalancing their portfolios annually, could have taken advantage of the market disequilibria by acquiring low P/E stocks. From the point of view of these investors, "market inefficiency" seems to have existed. Srinivasan (1997), Rao (1994) and Obaidullah (1990) examined the share price responses to announcement of dividend increase, bonus issue and equity rights and found that the Indian stock market is semi-strong form efficient. Chaturvedi (2000a, 200b) provided evidence for the market inefficiency. Raja et al. (2009) examined the informational efficiency of the Indian stock market in the semi-strong form of EMH and concluded that Indian stock market is efficient. However, Belgaumi (1995) studied the speed of adjustments of stock prices to half-yearly earnings announcements by examining the efficiency of Indian stock market. He concluded that learning lags existed in the Indian stock market and incorporation of publicly available information was slow. Therefore, Indian stock market is inefficient in the semi-strong form. Mallikarjunappa (2004), Iqbal and Mallikarjunappa (2007, 2008a, 2008b, 2010, 2011) and Iqbal, Mallikarjunappa and Nayak (2007) found that the Indian stock market does not react 
immediately to quarterly earnings announcements and provided an opportunity to earn abnormal returns. Therefore, they concluded that the Indian stock market is not efficient in the semi-strong form. Mallikarjunappa and Dsouza (2013, 2014), Saravanakumar and Mahadevan (2013), Seghal and Bijoy (2015) examined the semistrong form of efficient market hypothesis and found significant abnormal returns around the earnings announcement. The results showed that Indian market is predictable and therefore it exhibits market inefficiency.

The review of the studies shows that there is no clear evidence to accept that Indian stock market is efficient in semi-strong form. Therefore, an attempt is made to test semi-strong form of market efficiency in Indian stock market.

\section{Significance of the Study}

The EMH sates that dissemination of new information helps the traders to get fair pricing. Our study aims to empirically examine the stock price reaction to quarterly earnings news. The financial crisis of 2007-08 raised a big question mark on EMH theory. Jeremy Grantham who is a market strategist states that EMH is the pure reason for recent financial crisis. He felt that the financial thinkers had a "chronic

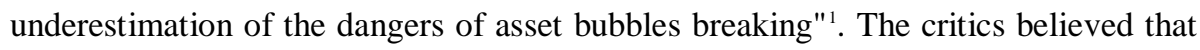
the corporates and the financial institutions are responsible for reduced market efficiency of financial markets. This happens because of development of new and complex products and creation of private information which reduces the accuracy. In this present scenario, it will be reasonable to test EMH by taking September 2012 quarter. The sub-prime lending crisis spread across the globe which caused global financial crisis had an impact on Indian stock market. During that period the FII had pulled out their investment from Indian market which caused stock market volatility and negative returns. Further, 2008 to 2011 was considered as post crisis period where the Indian market was regaining to a normal phase. In this study, we examined the market response immediately after the second phase (Post crisis) and therefore, we choose September 2012 quarter which will give true market picture as it is a normal phase in Indian stock market.

The presence of information technology brought the changes in market dynamics, price discovery and volatility in stock prices. The stock markets are working in the global scenario so as to raise the capital from the global investors. Any investors expect that stock market should be highly liquid and transparent. This will be achieved only when there is a proper and equal dissemination of information as and when there is new information flow in the market. The efficiency of the market is determined based on the speed in which the information is incorporated into security prices and not just with the speed with which it comes to the market. The market participant should get timely relevant information to make the decision which is still a question mark in emerging economies like India. There is more chance of insider trading and monopoly of information among group of people which leads to abnormal return. Therefore, there is a need to test the presence of information

${ }^{1}$ http://www.nytimes.com/2009/06/06/business/06nocera.html accessed on $17 / 12 / 2016$ at $8.30 \mathrm{AM}$. 
efficiency in this changed environment. Further it adds to the existing body of literature in EMH of emerging markets.

\section{Hypotheses of the Study}

The following hypotheses are proposed to be tested

1. The average abnormal return and cumulative average abnormal return are close to zero.

2. The average abnormal returns occur randomly.

3. There is no significant difference between the number of positive and negative average abnormal returns.

Event study methodology use average abnormal returns (AAR) and cumulative average abnormal returns (CAAR) of long period to capture overall reactions of the market during the corporate announcements (Fama 1991). This will help us to understand the idea about the average price behaviour in the market. The empirical evidences viz Brown and Warner (1980, 1985), Mallikarjunappa (2004) and Iqbal \& T. Mallikarjunappa (2007) showed that if AAR and CAAR are close to zero, market is efficient. The randomness in security prices are observed through Runs test (Campbell et al. 1997, Chiat and Finn 1983, Butler and Malaikah 1992 and Gujarati 2003). If the security prices are independent to each other, then the observed Runs are not significantly different from expected Runs and observed series are random. The sign test is based on the direction of the plus and minus sign of the AAR, and not on their numerical amount. If the probability value is greater than the significance level, we accept the null hypothesis (Mendenhall et al. (1989).

\section{Sample and Data}

The sample consists of BSE-500 based companies listed in Bombay Stock Exchange Limited (BSE). The main reason to select BSE-500 based companies is that, it covers 20 major industries in the Indian economy. This will give the true picture of the market and studying these companies will be more reasonable as they are reasonably best performing companies. The data comprises of dates of quarterly earnings announcements of September 2012 quarter. The dates of individual securities are collected for all BSE-500 companies based on availability. The final sample consists of 469 companies. We use daily closing prices of sample companies for the quarter and BSE-500 index is taken as market proxy. We classified the sample into three different portfolios based on net profit and net sales of current and corresponding quarters and the data is collected from Center for Monitoring Indian Economy (CMIE). If the percentage change in net profit and net sales is positive, it is referred as "good news" portfolio and if it is negative "bad news" portfolio and "overall portfolio" consist of all the sample companies. In the situation where firm's percentage changes in the net profit is positive and net sales is negative and vice versa, the sign of percentage change in the net profit is considered as a criterion to include that firm in the portfolio. Based on this, 248 companies formed as good news portfolio, 221 companies as bad news portfolio and 469 as full sample portfolio in this study. 


\section{Methodology}

Fama et al.(1969) developed the event study methodology to explore the information content available in stock splits and dividend announcements. Even today this methodology is widely accepted and extensively used in research all over the world. We have used quarterly earnings announcement news to examine the information content during the announcement by using event study methodology. The 61 days event window is used in the study. 30 days before the event and 30 days after the event are examined to see the relationship between information and return (i.e., $\mathrm{t}=$ $30, \ldots, 0, \ldots,+30)$. We have use 250 trading days as estimation period (i.e., $-280 \ldots-$ 31) which is also referred as non-event period in event study methodology. The three models; mean adjusted model, market adjusted model and market model are used to see the abnormal performance of the sample companies. Three models to see the reliability in findings of our study are used. The details of the models are given below.

\subsection{Abnormal Return Measures}

Let $R_{i, t}$ be the observed arithmetic return for security $\mathrm{i}$ on day $\mathrm{t}, A_{i, t}$ represents the abnormal return for security $\mathrm{i}$ on day $\mathrm{t}$. We use the following three models to estimate the abnormal return for each day in the event period.

\subsubsection{Mean Adjusted Model}

This model was initially developed by Masulis (1980). This model assumes that the expected return for the given security $\mathrm{i}$ is equal to constant $\bar{R}_{i}$. The abnormal return is equal to the difference between the actual return and expected return.

$$
\begin{gathered}
A_{i, t}=R_{i, t}-\bar{R}_{i} \\
\bar{R}_{i}=\frac{1}{250} \sum_{i=-280}^{-31} \bar{R}_{i, t}
\end{gathered}
$$

Where $A_{i, t}$ represents the abnormal return for security $\mathrm{i}$ on day $\mathrm{t}, \bar{R}_{i}$ is the average of security is daily returns in the estimation period $(-280,-31)$.

\subsubsection{Market Adjusted Model}

Under this model, the expected returns are equal across securities. The abnormal return is the difference between security return and market return and this model was developed by Cowles (1933) and Latane and Jones (1979).

$$
A_{i, t}=R_{i, t}-R_{m, t}
$$

Where $R_{m, t}$ is the return on the BSE-200 index for day $\mathrm{t}$

\subsubsection{OLS Market Model}

We use Sharpe (1964) market model where, we regress each security return with market return and use $\alpha$ and $\beta$ coefficients from simple regression to calculate expected return. The abnormal return is the difference between actual return and expected return of each security. The market model is given by 


$$
A_{i, t}=\alpha_{i}+\beta_{i} R_{m t}+e_{i t}
$$

where $\alpha_{i}$ and $\beta_{i}$ are OLS values from the estimation period.

The Beta is calculated using the following equation.

$$
\beta_{i}=\frac{N \sum_{t=1}^{N} R_{m t} R_{i t}-\left(\sum_{t=1}^{N} R_{m t}\right)\left(\sum_{t=1}^{N} R_{i t}\right)}{N\left(\sum_{t=1}^{N} R_{m t}^{2}\right)-N\left(\sum_{t=1}^{N} R_{m t}\right)^{2}}
$$

where, $\beta_{i}=$ slope of a straight line or beta coefficient of security ' $\mathrm{i}$ '. $R_{m t}=$ return on market index ' $\mathrm{m}$ ' during time period' $\mathrm{t}$ '. $R_{i t}=$ return on security ' $\mathrm{i}$ ' during time period' $t$ '. $N=$ number of observations.

The above three models were used by Brown and Warner (1980, pp. 207-209) to generate excess return. We compute the AARs (aggregates of abnormal returns for all $n$ securities) and CAARs (AAR is accumulated over a long period) based on this methodology. A number of other studies have also used this methodology. We expect that quarterly earnings impact the stock prices. To account for the general market movements, we fit an OLS that captures the price reactions due to market.

\subsection{Average Abnormal Returns (AAR)}

The following model is used to calculate average abnormal returns (AARs)

$$
A A R_{i t}=\frac{\sum_{i=1}^{N} A R_{i t}}{N}
$$

where, i represent different securities in the study; $\mathrm{N}=$ total number of securities. $\mathrm{t}=$ the days in the event window.

\subsection{The Cumulated Average Abnormal Return (CAAR)}

The AAR values are cumulated over 61-day period to find out cumulative average abnormal return (CAARs) and expect that the CAARs should be close to zero. The following formula is used for the CAARs

$$
C A A R_{t}=\sum_{t=-30}^{K} A A R_{i t}
$$

where $\mathrm{t}=-30, \ldots . .0, \ldots .+30$

\subsection{Standardized Abnormal Return (SAR) and Standardized Cumulative Average Abnormal Returns (SCAR)}

Standardized Abnormal Return (SAR) is calculated where, each excess return $A_{i}$, is first divided by its estimated standard deviation to yield a standardized excess return, $A^{\prime}{ }_{i, t}$. The standardized abnormal returns are then cumulated over time in order to ascertain standardized cumulative average abnormal returns (SCAR).

where

$$
A^{\prime}{ }_{i, t}=\frac{A_{i, t}}{\hat{S}\left(A_{i, t}\right)},
$$




$$
\begin{gathered}
\hat{S}\left(A_{i, t)}=\sqrt{\frac{\left(\sum_{t=-280}^{t=-31}\left(A_{i, t}-A_{i}^{*}\right)^{2}\right)}{249}},\right. \\
A^{*}{ }_{i}=\frac{1}{250} \sum_{t=-280}^{t=-31} A_{i, t}
\end{gathered}
$$

The test statistics for any given day $(\mathrm{t}=0)$ is calculated as

$$
\left(\sum_{i=1}^{N_{t}} A_{j, t}^{\prime}\right) \cdot\left(N_{t}\right)^{-\frac{1}{2}}
$$

where $\mathrm{N}=$ the number of sample securities at day $\mathrm{t}$.

\subsection{Parametric Significance Test}

Parametric $t$ test is used to assess the significance of AARs and CAARs. The 5\% level of significance with appropriate degree of freedom is used to test the null hypothesis that there are no significant abnormal returns after the event day. It is assumed that if the market is efficient, AARs and CAARs values should be close to zero.

\subsubsection{The $t$ Test Statistic for AARs}

This statistic is given by

$$
t=\frac{A A R}{\sigma(A A R)}
$$

where $\mathrm{AAR}=$ average abnormal return, $\sigma(A A R)=$ standard error of average abnormal return.

The standard error is calculated by using following formula.

$$
S . E=\frac{\sigma}{\sqrt{n}}
$$

where, $\mathrm{S} . \mathrm{E}=$ standard error,$\sigma=$ standard deviation, $\mathrm{n}=$ number of observation

\subsubsection{The $t$ Test Statistic for CAARs}

This statistic is given by

$$
t=\frac{C A A R}{\sigma(C A A R)}
$$

where, $\sigma(\mathrm{CAAR})$ is the standard error of cumulative average abnormal return. The standard error is calculated by using the following formula

$$
S . E=\frac{\sigma}{\sqrt{n}}
$$


$\mathrm{S} . \mathrm{E}=$ standard error, $\sigma=$ standard deviation, $\mathrm{n}=$ number of observations.

\subsection{Non-Parametric Significance Test}

In addition to t test, non-parametric tests like, Runs and Sign tests are used to test the hypotheses.

\subsubsection{Runs Test}

This test was developed by Levene (1952) to analyze the randomness in the behavior of observed numbers. In this paper Runs test on AARs before and after the event day and also for the entire event window to test for the randomness in the occurrence of AARs is applied.

The Runs test is calculated by using the following formula.

$$
\mu_{r}=\left(\frac{2 n_{1} n_{2}}{n_{1}+n_{2}}\right)+1
$$

where, $\mu_{r}=$ mean number of runs, $\mathrm{n}_{1}=$ number of positive AARs, $\mathrm{n}_{2}=$ number of negative AARs, $r=$ number of runs (actual sequence of counts)

The standard error of the expected number of runs can be calculated by using following formula.

$$
\sigma_{\mathrm{r}}=\sqrt{\frac{2 \mathrm{n}_{1} \mathrm{n}_{2}\left(2 \mathrm{n}_{1} \mathrm{n}_{2}-\mathrm{n}_{1}-\mathrm{n}_{2}\right)}{\left(\mathrm{n}_{1}+\mathrm{n}_{2}\right)^{2}\left(\mathrm{n}_{1}+\mathrm{n}_{2}-1\right)}}
$$

The difference between actual and expected number of the runs is calculated as

$$
\mathrm{Z}=\frac{r-\mu_{r}}{\sigma_{r}}
$$

\subsubsection{Sign Test}

Mendenhall et al. (1989) developed Sign test which considers positive and negative signs instead of quantitative values. The null hypothesis for this test is that there is no significant difference between the number of positive and negative AARs. We apply Sign test statistics before and after the event day and also for the event window. The standard error is computed using the following formula:

$$
\sigma_{p}=\sqrt{\frac{p q}{n}}
$$

Where, $\sigma_{p}=$ standard error of the proportion, $\mathrm{p}=$ expected proportion of positive $\mathrm{AAR}=0.5, \mathrm{q}=$ expected proportion of negative $\mathrm{AAR}=0.5, \mathrm{n}=$ number of AAR To compute the value of Sign test we use the following equation

$$
\mathrm{Z}=\frac{\bar{P}-P_{H 0}}{\sigma_{P}}
$$

$\bar{p}=$ actual proportion of AAR in the respective quarters having positive signs. $P_{H O}=$ hypothesized proportion 0.5 


\subsection{Cohen et al. (1983) Methodology}

Cohen et al. (1983) to see the price adjustment process during the earnings announcement was adopted. Market model regression technique was used and applied for sample companies using 20 return intervals spanning one to twenty days for both pre and post-event data. This provides $i^{*} 20 * 2$ estimates of betas. BSE-500 index is used as proxy to calculate market return.

$R_{i j k t}=\alpha_{i j k}+\beta_{i j k} R_{m j k t}+e_{i j k t} \mathrm{j}=1 \ldots .20, \mathrm{i}=1 \ldots \ldots \mathrm{nk}=1,2$

where, $R_{i j k t}$ is the return to stock $i$ on day $t$, for return interval $j$, using the $k$ sample periods ( $k$ has a value of 1 in the pre-event period and has a value of 2 in the postevent period). $R_{m j k t}$ is the market return on day $t$, using interval $j$ and sample $k$. According to Schwartz (1991), the first-pass beta is expected to reach its true value asymptotically as the measurement interval, L, approaches infinity. To test this expectation, we used the 40 (pre and post event) first-pass market model regression beta estimates $\left(b_{j, 1 L E}\right)$ for each stock to run the second-pass, stock-specific regression.

$b_{j, 1 L E}=a_{j, 2}+b_{j, 2} \ln \left(1+L^{-1}\right)+C_{j, 2}\left(\right.$ Dummy $\left._{j E} * \ln \left(1+L^{-1}\right)\right)+e_{j L H}$

where $b_{j, 1 L E}$ is the first-pass beta estimate for security $\mathrm{j}$ based on L-day stock returns for the time period $\mathrm{E}$; and denotes either the period before or after the event; $a_{j, 2}, b_{j, 2}$ , and $C_{j, 2}$ are second-pass parameter estimates, $\mathrm{L}$ is the length of the holding period, in days, for which the stock returns were calculated; Dummy $y_{j E}$ is a binary variable equal to one if the first-pass beta is estimated using the post-event data and zero if the first-pass beta is estimated using the pre-event data and $e_{j L H}$ is a stochastic disturbance term. The event study tests are operationalized by an interaction variable that equals $1 * \ln \left(1+L^{-1}\right)$ for the post-event period and zero for the pre-event period. This variable is included in the above equation to capture any changes in the relation between $\mathrm{L}$ and the first-pass betas after the quarterly earnings announcement.

Apart from this, we use $\mathrm{R}$ square which is influencing by the choice of return intervals. R-square is an indicator of information quality and want to see whether low R-square indicate early resolution of uncertainty through the arrival of firmspecific information, or does it indicate a high level of uncertainty that remains unresolved. The low R-square firms have lower future earnings response coefficient, indicating that their current stock price incorporates a smaller amount of future earnings news.

\section{Results and Analysis}

Table 1 and Figure 1 shows the AAR and CAAR values of full sample earnings announcement of mean adjusted model, market adjusted model and market model of September -2012 quarter. The significant AARs are observed for mean adjusted model and market model for most of the days in the event window of overall portfolio. In the case of market adjusted model, AARs are positive and insignificant for majority of the days surrounding the event window. This shows that market is positively responded to the earnings announcement news. Further, the AAR on the 
event day (day 0) for all the three models are negative and insignificant. It shows that quarterly earnings announcement had a negative impact on the market on the day of announcement. In the pre-event period, AARs are significantly positive continuously from day -30 to -20 and from day 14 to 25 of post event period. This result implies that the investors are able to anticipate the information by understanding some financial variables and try to generate some abnormal returns. On pre and post earnings announcement basis, positively significant CAARs are found throughout the event window. This shows that the investors can hold their investment during the earnings announcement and generate abnormal profits which is against the theory of EMH. These results prove that earnings announcement news is a predictable event. We used three models to see the consistency in the results. Out of the three, two models viz mean adjusted model and market model generated similar results.

Table $1 A A R$ and CAAR values of Full Sample Earnings Announcements

\begin{tabular}{|c|c|c|c|c|c|c|c|c|c|c|c|c|}
\hline \multirow[t]{2}{*}{ Da } & \multicolumn{4}{|c|}{ Mean Adjusted Model } & \multicolumn{4}{|c|}{ Market Adjusted Model } & \multicolumn{4}{|c|}{ Market model } \\
\hline & AAAR & $\begin{array}{c}\mathbf{t} \\
\text { value }\end{array}$ & $\begin{array}{c}\text { CAA } \\
\mathbf{R}\end{array}$ & $t$ value & AAR & $\begin{array}{c}\mathbf{t} \\
\text { value }\end{array}$ & $\begin{array}{c}\text { CAA } \\
\mathbf{R}\end{array}$ & $\begin{array}{c}\mathbf{t} \\
\text { value }\end{array}$ & AAR & $\begin{array}{c}\mathbf{t} \\
\text { value }\end{array}$ & $\begin{array}{c}\text { CAA } \\
\mathbf{R}\end{array}$ & t value \\
\hline-30 & $\begin{array}{l}0.25 \\
548\end{array}$ & $\begin{array}{c}5.851 \\
45^{*}\end{array}$ & $\begin{array}{c}0.25 \\
548\end{array}$ & $\begin{array}{c}5.8514 \\
5^{*}\end{array}$ & $\begin{array}{l}0.09 \\
378\end{array}$ & $\begin{array}{c}1.900 \\
06\end{array}$ & $\begin{array}{c}0.09 \\
378\end{array}$ & 06 & $\begin{array}{l}0.30 \\
013\end{array}$ & $\begin{array}{c}6.775 \\
28^{*}\end{array}$ & $\begin{array}{c}0.30 \\
013\end{array}$ & $8 * 752$ \\
\hline-29 & $\begin{array}{l}0.15 \\
965\end{array}$ & $\begin{array}{c}3.690 \\
62 *\end{array}$ & $\begin{array}{c}0.41 \\
513\end{array}$ & $\begin{array}{c}6.7858 \\
7 *\end{array}$ & $\begin{array}{c}0.02 \\
508\end{array}$ & $\begin{array}{c}0.533 \\
56\end{array}$ & $\begin{array}{l}0.11 \\
887\end{array}$ & $\begin{array}{c}1.787 \\
97\end{array}$ & $\begin{array}{l}0.20 \\
385\end{array}$ & $\begin{array}{c}4.664 \\
89^{*}\end{array}$ & $\begin{array}{l}0.50 \\
399\end{array}$ & $\begin{array}{c}8.1550 \\
8^{*}\end{array}$ \\
\hline-28 & $\begin{array}{l}0.25 \\
766\end{array}$ & $\begin{array}{c}5.853 \\
07^{*}\end{array}$ & $\begin{array}{l}0.67 \\
279\end{array}$ & $\begin{array}{c}8.8238 \\
1 *\end{array}$ & $\begin{array}{l}0.00 \\
107\end{array}$ & $\begin{array}{c}0.021 \\
67\end{array}$ & $\begin{array}{l}0.11 \\
780\end{array}$ & $\begin{array}{c}1.376 \\
36\end{array}$ & $\begin{array}{l}0.29 \\
060\end{array}$ & $\begin{array}{c}6.494 \\
40^{*}\end{array}$ & $\begin{array}{l}0.79 \\
459\end{array}$ & $\begin{array}{c}10.252 \\
38^{*}\end{array}$ \\
\hline-27 & $\begin{array}{l}0.15 \\
201 \\
\end{array}$ & $\begin{array}{c}3.426 \\
55^{*}\end{array}$ & $\begin{array}{l}0.82 \\
480\end{array}$ & $\begin{array}{c}9.2963 \\
0^{*}\end{array}$ & $\begin{array}{l}0.06 \\
878\end{array}$ & $\begin{array}{c}1.481 \\
27\end{array}$ & $\begin{array}{l}0.04 \\
902\end{array}$ & $\begin{array}{c}0.527 \\
90\end{array}$ & $\begin{array}{l}0.18 \\
428\end{array}$ & $\begin{array}{c}4.173 \\
48^{*}\end{array}$ & $\begin{array}{l}0.97 \\
887\end{array}$ & $\begin{array}{c}11.084 \\
23 *\end{array}$ \\
\hline-26 & $\begin{array}{l}0.21 \\
247\end{array}$ & $\begin{array}{c}4.500 \\
19^{*}\end{array}$ & $\begin{array}{l}1.03 \\
727\end{array}$ & $\begin{array}{c}9.8250 \\
4^{*}\end{array}$ & $\begin{array}{l}0.01 \\
810\end{array}$ & $\begin{array}{c}0.364 \\
02\end{array}$ & $\begin{array}{l}0.06 \\
712\end{array}$ & $\begin{array}{c}0.603 \\
64\end{array}$ & $\begin{array}{l}0.25 \\
481\end{array}$ & $\begin{array}{c}5.566 \\
29^{*}\end{array}$ & $\begin{array}{l}1.23 \\
369\end{array}$ & $\begin{array}{c}12.052 \\
07^{*}\end{array}$ \\
\hline-25 & $\begin{array}{l}0.16 \\
898\end{array}$ & $\begin{array}{c}3.991 \\
79^{*}\end{array}$ & $\begin{array}{l}1.20 \\
626\end{array}$ & $\begin{array}{c}11.633 \\
04 *\end{array}$ & $\begin{array}{l}0.07 \\
399\end{array}$ & $\begin{array}{c}1.607 \\
58\end{array}$ & $\begin{array}{c}0.14 \\
112\end{array}$ & $\begin{array}{c}1.251 \\
64\end{array}$ & $\begin{array}{l}0.20 \\
844\end{array}$ & $\begin{array}{c}4.848 \\
56^{*}\end{array}$ & $\begin{array}{l}1.44 \\
213\end{array}$ & $\begin{array}{c}13.694 \\
70^{*}\end{array}$ \\
\hline-24 & $\begin{array}{l}0.31 \\
943\end{array}$ & $\begin{array}{c}6.542 \\
22^{*}\end{array}$ & $\begin{array}{l}1.52 \\
569\end{array}$ & $\begin{array}{c}11.810 \\
42 *\end{array}$ & $\begin{array}{c}0.03 \\
449\end{array}$ & $\begin{array}{c}0.646 \\
90\end{array}$ & $\begin{array}{l}0.17 \\
561\end{array}$ & $\begin{array}{c}1.244 \\
98\end{array}$ & $\begin{array}{l}0.35 \\
652\end{array}$ & $\begin{array}{c}7.268 \\
55^{*}\end{array}$ & $\begin{array}{l}1.79 \\
866\end{array}$ & $\begin{array}{c}13.859 \\
81^{*}\end{array}$ \\
\hline-23 & $\begin{array}{l}0.26 \\
786\end{array}$ & $\begin{array}{c}6.158 \\
25^{*}\end{array}$ & $\begin{array}{l}1.79 \\
354\end{array}$ & $\begin{array}{c}14.578 \\
73^{*}\end{array}$ & $\begin{array}{l}0.10 \\
611\end{array}$ & $\begin{array}{c}2.276 \\
70^{*}\end{array}$ & $\begin{array}{c}0.28 \\
172\end{array}$ & $\begin{array}{c}2.137 \\
04 *\end{array}$ & $\begin{array}{l}0.29 \\
791\end{array}$ & $\begin{array}{c}6.749 \\
25^{*}\end{array}$ & $\begin{array}{l}2.09 \\
657\end{array}$ & $\begin{array}{c}16.793 \\
07^{*}\end{array}$ \\
\hline-22 & $\begin{array}{l}0.17 \\
563\end{array}$ & $\begin{array}{c}3.922 \\
46^{*}\end{array}$ & $\begin{array}{l}1.96 \\
918\end{array}$ & $\begin{array}{c}14.659 \\
34 *\end{array}$ & $\begin{array}{l}0.06 \\
094\end{array}$ & $\begin{array}{c}1.298 \\
01\end{array}$ & $\begin{array}{l}0.34 \\
266\end{array}$ & $\begin{array}{c}2.432 \\
91 *\end{array}$ & $\begin{array}{c}0.20 \\
841\end{array}$ & $\begin{array}{c}4.562 \\
65^{*}\end{array}$ & $\begin{array}{c}2.30 \\
498\end{array}$ & $\begin{array}{c}16.820 \\
74^{*}\end{array}$ \\
\hline-21 & $\begin{array}{l}0.11 \\
944\end{array}$ & $\begin{array}{c}2.872 \\
38^{*}\end{array}$ & $\begin{array}{l}2.08 \\
862\end{array}$ & $\begin{array}{c}15.883 \\
23^{*}\end{array}$ & $\begin{array}{l}0.07 \\
038\end{array}$ & $\begin{array}{c}1.606 \\
57\end{array}$ & $\begin{array}{l}0.41 \\
303\end{array}$ & $\begin{array}{c}2.981 \\
64 *\end{array}$ & $\begin{array}{l}0.15 \\
750\end{array}$ & $\begin{array}{c}3.588 \\
24 *\end{array}$ & $\begin{array}{l}2.46 \\
248\end{array}$ & $\begin{array}{c}17.740 \\
58^{*}\end{array}$ \\
\hline-20 & $\begin{array}{l}0.08 \\
722\end{array}$ & $\begin{array}{c}1.985 \\
35^{*}\end{array}$ & $\begin{array}{l}2.17 \\
584\end{array}$ & $\begin{array}{c}14.933 \\
64^{*}\end{array}$ & $\begin{array}{c}0.01 \\
448\end{array}$ & $\begin{array}{c}0.315 \\
91\end{array}$ & $\begin{array}{l}0.42 \\
751\end{array}$ & $\begin{array}{c}2.812 \\
71^{*}\end{array}$ & $\begin{array}{c}0.11 \\
960\end{array}$ & $\begin{array}{c}2.640 \\
01 *\end{array}$ & $\begin{array}{l}2.58 \\
208\end{array}$ & $\begin{array}{c}17.185 \\
55^{*}\end{array}$ \\
\hline-19 & $\begin{array}{l}0.09 \\
706\end{array}$ & $\begin{array}{c}1.933 \\
57\end{array}$ & $\begin{array}{l}2.27 \\
290\end{array}$ & $\begin{array}{c}13.071 \\
08^{*}\end{array}$ & $\begin{array}{l}0.03 \\
148 \\
\end{array}$ & $\begin{array}{c}0.635 \\
88 \\
\end{array}$ & $\begin{array}{c}0.39 \\
603\end{array}$ & $\begin{array}{c}2.309 \\
35^{*}\end{array}$ & $\begin{array}{l}0.13 \\
381\end{array}$ & $\begin{array}{c}2.598 \\
42^{*} \\
\end{array}$ & $\begin{array}{l}2.71 \\
589 \\
\end{array}$ & $\begin{array}{c}15.224 \\
23^{*}\end{array}$ \\
\hline-18 & $\begin{array}{l}0.15 \\
952\end{array}$ & $\begin{array}{c}3.632 \\
09^{*}\end{array}$ & $\begin{array}{l}2.43 \\
242\end{array}$ & $\begin{array}{c}15.360 \\
57^{*}\end{array}$ & $\begin{array}{c}0.07 \\
038\end{array}$ & $\begin{array}{c}1.590 \\
56\end{array}$ & $\begin{array}{c}0.46 \\
641\end{array}$ & $\begin{array}{c}2.923 \\
33^{*}\end{array}$ & $\begin{array}{l}0.19 \\
163\end{array}$ & $\begin{array}{c}4.217 \\
41^{*}\end{array}$ & $\begin{array}{l}2.90 \\
753\end{array}$ & $\begin{array}{c}17.746 \\
92^{*}\end{array}$ \\
\hline
\end{tabular}




\begin{tabular}{|c|c|c|c|c|c|c|c|c|c|c|c|c|}
\hline-17 & $\begin{array}{l}0.14 \\
255\end{array}$ & $\begin{array}{c}3.622 \\
76^{*}\end{array}$ & $\begin{array}{l}2.57 \\
497\end{array}$ & $\begin{array}{l}7.489 \\
90^{*}\end{array}$ & $\begin{array}{c}0.11 \\
019\end{array}$ & $\begin{array}{l}2.546 \\
22^{*}\end{array}$ & $\begin{array}{l}0.57 \\
661\end{array}$ & $\begin{array}{c}3.560 \\
86^{*}\end{array}$ & $\begin{array}{l}0.18 \\
368\end{array}$ & $\begin{array}{l}4.608 \\
87^{*}\end{array}$ & $\begin{array}{l}3.09 \\
120\end{array}$ & $\begin{array}{c}20.730 \\
18^{*}\end{array}$ \\
\hline-16 & 254 & $\begin{array}{c}1.082 \\
55\end{array}$ & $\begin{array}{l}2.61 \\
750\end{array}$ & $\begin{array}{l}7.199 \\
34^{*}\end{array}$ & $\begin{array}{l}0.03 \\
123\end{array}$ & $\begin{array}{c}0.776 \\
69\end{array}$ & $\begin{array}{c}0.54 \\
537\end{array}$ & $\begin{array}{l}3.501 \\
64 *\end{array}$ & $\begin{array}{c}0.09 \\
119\end{array}$ & $67^{*}$ & $\begin{array}{l}3.18 \\
239\end{array}$ & $\begin{array}{l}20.506 \\
18^{*}\end{array}$ \\
\hline-15 & $\begin{array}{c}0.00 \\
994\end{array}$ & $\begin{array}{c}0.245 \\
71 \\
\end{array}$ & $\begin{array}{l}2.60 \\
756\end{array}$ & $\begin{array}{l}16.109 \\
84^{*}\end{array}$ & $\begin{array}{l}0.03 \\
666\end{array}$ & $\begin{array}{c}0.898 \\
31\end{array}$ & $\begin{array}{l}0.58 \\
203\end{array}$ & $\begin{array}{c}3.565 \\
74^{*}\end{array}$ & $\begin{array}{l}0.01 \\
578\end{array}$ & $\begin{array}{l}.372 \\
42 \\
\end{array}$ & $\begin{array}{l}3.19 \\
817\end{array}$ & $\begin{array}{l}18.866 \\
96^{*} \\
\end{array}$ \\
\hline-14 & $\begin{array}{l}0.01 \\
137\end{array}$ & $\begin{array}{c}0.298 \\
56\end{array}$ & $\begin{array}{l}2.61 \\
893\end{array}$ & $\begin{array}{l}6.674 \\
23^{*}\end{array}$ & $\begin{array}{l}0.02 \\
310\end{array}$ & $\begin{array}{c}0.544 \\
36\end{array}$ & $\begin{array}{c}0.60 \\
513\end{array}$ & $\begin{array}{c}3.458 \\
17^{*}\end{array}$ & .04 & $\begin{array}{l}1.255 \\
07\end{array}$ & $\begin{array}{l}3.24 \\
810\end{array}$ & $\begin{array}{l}19.804 \\
77^{*}\end{array}$ \\
\hline-13 & 254 & $67 *$ & & $36^{*}$ & $\begin{array}{l}0.03 \\
880\end{array}$ & $\begin{array}{l}0.891 \\
08\end{array}$ & & $13^{*}$ & & & .40 & $\begin{array}{l}18.425 \\
55^{*}\end{array}$ \\
\hline-12 & $\begin{array}{c}- \\
0.01 \\
279 \\
\end{array}$ & $\begin{array}{c}- \\
0.294 \\
07\end{array}$ & & $\begin{array}{c}14.337 \\
08^{*}\end{array}$ & $\begin{array}{l}0.02 \\
942\end{array}$ & $\begin{array}{l}.670 \\
52\end{array}$ & $\begin{array}{l}0.67 \\
335\end{array}$ & $\begin{array}{c}3.520 \\
34^{*}\end{array}$ & & $\begin{array}{c}0.738 \\
42\end{array}$ & $\begin{array}{l}3.43 \\
645\end{array}$ & $\begin{array}{c}17.521 \\
49^{*}\end{array}$ \\
\hline-11 & & $\begin{array}{c}1.771 \\
54\end{array}$ & & $39^{*}$ & & $\begin{array}{c}0.665 \\
93\end{array}$ & & & & & $\begin{array}{l}.56 \\
49\end{array}$ & $\begin{array}{l}16.538 \\
99^{*}\end{array}$ \\
\hline-10 & 292 & 40 & & $02 *$ & 555 & 09 & & $18^{*}$ & & 88 & $\begin{array}{c}3.60 \\
186\end{array}$ & $59^{*}$ \\
\hline-9 & $\begin{array}{r}0.01 \\
462 \\
\end{array}$ & $\begin{array}{c}- \\
0.386 \\
48 \\
\end{array}$ & $\begin{array}{l}2.79 \\
070\end{array}$ & $\begin{array}{c}15.732 \\
01 *\end{array}$ & $\begin{array}{c}- \\
0.02 \\
619 \\
\end{array}$ & $\begin{array}{c}- \\
0.657 \\
99 \\
\end{array}$ & $\begin{array}{l}0.72 \\
391\end{array}$ & $\begin{array}{c}3.876 \\
82^{*}\end{array}$ & & 23 & $\begin{array}{l}3.62 \\
099\end{array}$ & $\begin{array}{c}19.223 \\
17^{*}\end{array}$ \\
\hline-8 & $\begin{array}{c}0.17 \\
059 \\
\end{array}$ & $\begin{array}{c}- \\
4.293 \\
63 *\end{array}$ & & $\begin{array}{c}13.750 \\
70^{*}\end{array}$ & $\begin{array}{c}0.06 \\
968\end{array}$ & $\begin{array}{c}1.635 \\
08 \\
\end{array}$ & & $\begin{array}{c}3.201 \\
03^{*}\end{array}$ & $\begin{array}{l}0.13 \\
629 \\
\end{array}$ & $-\overline{203}$ & $\begin{array}{l}.48 \\
70\end{array}$ & $\begin{array}{c}17.077 \\
14^{*}\end{array}$ \\
\hline-7 & $\begin{array}{c}0.00 \\
041\end{array}$ & $\begin{array}{c}0.009 \\
86\end{array}$ & & & & $\begin{array}{c}0.863 \\
58\end{array}$ & & $\begin{array}{c}3.291 \\
65^{*}\end{array}$ & & $\begin{array}{c}0.840 \\
39\end{array}$ & $\begin{array}{c}3.52 \\
170\end{array}$ & $\begin{array}{c}16.325 \\
15^{*}\end{array}$ \\
\hline-6 & 111 & $79 *$ & & $26^{*}$ & & 77 & & $15^{*}$ & & $05^{*}$ & $\begin{array}{l}3.64 \\
809\end{array}$ & $12^{*}$ \\
\hline-5 & & $\begin{array}{r}2.01 \\
50\end{array}$ & $\begin{array}{c}2.62 \\
620\end{array}$ & $\begin{array}{c}13.789 \\
38^{*}\end{array}$ & & $\begin{array}{c}1.885 \\
42 \\
\end{array}$ & $\begin{array}{c}0.62 \\
529\end{array}$ & $\begin{array}{c}3.110 \\
01^{*}\end{array}$ & & $\begin{array}{c}0.837 \\
00\end{array}$ & $\begin{array}{l}3.61 \\
469\end{array}$ & $\begin{array}{c}17.767 \\
47^{*}\end{array}$ \\
\hline-4 & & $\begin{array}{c}0.372 \\
31 \\
\end{array}$ & & $38 *$ & & $\begin{array}{c}0.849 \\
96 \\
\end{array}$ & & $\begin{array}{c}2.998 \\
71^{*}\end{array}$ & & 93 & $\begin{array}{l}63 \\
94\end{array}$ & $95^{*}$ \\
\hline-3 & $\begin{array}{l}0.05 \\
061\end{array}$ & $\begin{array}{c}1.366 \\
09\end{array}$ & & $\begin{array}{c}13.583 \\
33^{*}\end{array}$ & & $\begin{array}{c}1.930 \\
76\end{array}$ & & $\begin{array}{c}3.228 \\
03^{*}\end{array}$ & & & $\begin{array}{l}.72 \\
53\end{array}$ & $\begin{array}{c}17.782 \\
63^{*}\end{array}$ \\
\hline-2 & $\begin{array}{c}0.14 \\
894\end{array}$ & $30 *$ & 186 & $88 *$ & & 99 & & $12^{*}$ & & $85^{*}$ & $\begin{array}{l}3.90 \\
312\end{array}$ & $23^{*}$ \\
\hline-1 & $\begin{array}{c}0.04 \\
169\end{array}$ & $\begin{array}{c}1.091 \\
48\end{array}$ & & $\begin{array}{c}13.639 \\
03 *\end{array}$ & & $\begin{array}{c}1.977 \\
47^{*}\end{array}$ & & $\begin{array}{c}3.790 \\
71^{*}\end{array}$ & & & $\begin{array}{l}3.98 \\
732\end{array}$ & $\begin{array}{c}18.528 \\
52 *\end{array}$ \\
\hline 0 & $\begin{array}{c}- \\
0.12 \\
096\end{array}$ & $\begin{array}{c}- \\
1.906 \\
83 \\
\end{array}$ & $\begin{array}{l}2.73 \\
259\end{array}$ & $\begin{array}{l}73 \\
0 *\end{array}$ & $\begin{array}{c}- \\
0.06 \\
850 \\
\end{array}$ & $\begin{array}{c}- \\
1.028 \\
09\end{array}$ & & $\begin{array}{c}2.003 \\
19^{*}\end{array}$ & $\begin{array}{c}- \\
0.09 \\
725 \\
\end{array}$ & $\begin{array}{c}- \\
1.504 \\
73 \\
\end{array}$ & $\begin{array}{l}89 \\
97\end{array}$ & $68^{*}$ \\
\hline 1 & $\begin{array}{c}0.06 \\
526\end{array}$ & $\begin{array}{c}1.052 \\
07\end{array}$ & $\begin{array}{l}2.79 \\
785\end{array}$ & $\begin{array}{c}7.9736 \\
6^{*}\end{array}$ & $\begin{array}{l}0.05 \\
895\end{array}$ & $\begin{array}{c}0.902 \\
82\end{array}$ & & $\begin{array}{c}2.171 \\
48^{*}\end{array}$ & & $\begin{array}{c}1.484 \\
99\end{array}$ & $\begin{array}{l}3.98 \\
458\end{array}$ & $\begin{array}{c}11.068 \\
30^{*}\end{array}$ \\
\hline 2 & $\begin{array}{l}0.03 \\
489\end{array}$ & $\begin{array}{c}0.922 \\
90\end{array}$ & 2.83 & $\begin{array}{c}13.043 \\
74 *\end{array}$ & $\begin{array}{c}- \\
0.02 \\
412 \\
\end{array}$ & $\begin{array}{c}0.590 \\
31 \\
\end{array}$ & & $\begin{array}{c}3.314 \\
78^{*}\end{array}$ & & $\begin{array}{c}1.717 \\
41\end{array}$ & $\begin{array}{l}4.05 \\
212\end{array}$ & $\begin{array}{c}17.934 \\
40^{*}\end{array}$ \\
\hline 3 & $\begin{array}{l}0.02 \\
919\end{array}$ & & 355 & $99 *$ & $\begin{array}{c}0.03 \\
472\end{array}$ & $\begin{array}{c}0.867 \\
89\end{array}$ & 264 & $\begin{array}{c}3.484 \\
03^{*}\end{array}$ & 582 & 91 & $\begin{array}{l}4.05 \\
794\end{array}$ & $\begin{array}{c}17.447 \\
30 *\end{array}$ \\
\hline
\end{tabular}




\begin{tabular}{|c|c|c|c|c|c|c|c|c|c|c|c|c|}
\hline 4 & $\begin{array}{l}0.04 \\
207 \\
\end{array}$ & $\begin{array}{c}1.091 \\
48 \\
\end{array}$ & $\begin{array}{c}2.76 \\
148\end{array}$ & $\begin{array}{c}12.110 \\
10^{*}\end{array}$ & $\begin{array}{l}0.02 \\
230\end{array}$ & $\begin{array}{c}0.554 \\
80\end{array}$ & $\begin{array}{l}0.83 \\
494\end{array}$ & $\begin{array}{c}3.510 \\
50^{*}\end{array}$ & $\begin{array}{l}0.00 \\
887 \\
\end{array}$ & $\begin{array}{c}0.220 \\
90 \\
\end{array}$ & $\begin{array}{l}4.04 \\
907\end{array}$ & $\begin{array}{c}17.039 \\
35^{*}\end{array}$ \\
\hline 5 & $\begin{array}{c}0.00 \\
949\end{array}$ & $\begin{array}{c}0.249 \\
21\end{array}$ & $\begin{array}{l}2.75 \\
199\end{array}$ & $\begin{array}{c}12.042 \\
39^{*}\end{array}$ & $\begin{array}{l}0.00 \\
518\end{array}$ & $\begin{array}{c}0.133 \\
67\end{array}$ & $\begin{array}{l}0.84 \\
012\end{array}$ & $\begin{array}{c}3.614 \\
77^{*}\end{array}$ & $\begin{array}{l}0.02 \\
761\end{array}$ & $\begin{array}{c}0.680 \\
58\end{array}$ & $\begin{array}{l}4.07 \\
668\end{array}$ & $\begin{array}{c}16.746 \\
48^{*}\end{array}$ \\
\hline 6 & $\begin{array}{c}- \\
0.01 \\
827\end{array}$ & $\begin{array}{c}- \\
0.501 \\
41\end{array}$ & $\begin{array}{l}2.73 \\
372\end{array}$ & $\begin{array}{c}12.333 \\
94^{*}\end{array}$ & $\begin{array}{l}0.00 \\
922\end{array}$ & $\begin{array}{c}0.244 \\
26\end{array}$ & $\begin{array}{c}0.84 \\
934\end{array}$ & $\begin{array}{l}3.697 \\
93 *\end{array}$ & $\begin{array}{l}0.01 \\
867\end{array}$ & $\begin{array}{c}0.477 \\
47\end{array}$ & $\begin{array}{l}4.09 \\
535\end{array}$ & $\begin{array}{c}17.221 \\
26^{*}\end{array}$ \\
\hline 7 & $\begin{array}{l}0.07 \\
715 \\
\end{array}$ & $\begin{array}{c}2.197 \\
62^{*} \\
\end{array}$ & $\begin{array}{l}2.65 \\
657\end{array}$ & $\begin{array}{c}12.276 \\
24^{*}\end{array}$ & $\begin{array}{l}0.03 \\
273 \\
\end{array}$ & $\begin{array}{c}0.902 \\
78\end{array}$ & $\begin{array}{l}0.81 \\
662\end{array}$ & $\begin{array}{c}3.654 \\
33^{*}\end{array}$ & $\begin{array}{l}0.03 \\
385\end{array}$ & $\begin{array}{c}0.919 \\
79\end{array}$ & $\begin{array}{l}4.06 \\
150\end{array}$ & $\begin{array}{c}17.901 \\
09^{*}\end{array}$ \\
\hline 8 & $\begin{array}{l}0.02 \\
358\end{array}$ & $\begin{array}{c}- \\
0.595 \\
76\end{array}$ & $\begin{array}{l}2.63 \\
300\end{array}$ & $\begin{array}{c}10.653 \\
75^{*}\end{array}$ & $\begin{array}{c}- \\
0.03 \\
210\end{array}$ & $\begin{array}{c}- \\
0.792 \\
58\end{array}$ & $\begin{array}{c}0.78 \\
452\end{array}$ & $\begin{array}{c}3.101 \\
81 *\end{array}$ & $\begin{array}{l}0.01 \\
265\end{array}$ & $\begin{array}{c}0.306 \\
72\end{array}$ & $\begin{array}{l}4.07 \\
415\end{array}$ & $\begin{array}{c}15.815 \\
85^{*}\end{array}$ \\
\hline 9 & $\begin{array}{c}0.08 \\
879\end{array}$ & $\begin{array}{c}2.151 \\
71^{*}\end{array}$ & $\begin{array}{l}2.72 \\
179\end{array}$ & $\begin{array}{c}10.428 \\
66^{*}\end{array}$ & $\begin{array}{l}0.05 \\
228\end{array}$ & $\begin{array}{c}1.237 \\
64\end{array}$ & $\begin{array}{c}0.83 \\
679\end{array}$ & $\begin{array}{c}3.132 \\
35^{*}\end{array}$ & $\begin{array}{c}0.12 \\
109\end{array}$ & $\begin{array}{c}2.856 \\
62^{*}\end{array}$ & $\begin{array}{l}4.19 \\
524\end{array}$ & $\begin{array}{c}15.648 \\
21^{*}\end{array}$ \\
\hline 10 & $\begin{array}{l}0.09 \\
179\end{array}$ & $\begin{array}{c}1.792 \\
08\end{array}$ & $\begin{array}{l}2.81 \\
358\end{array}$ & $\begin{array}{c}8.5790 \\
9^{*}\end{array}$ & $\begin{array}{l}0.02 \\
023\end{array}$ & $\begin{array}{c}0.400 \\
46\end{array}$ & $\begin{array}{l}0.85 \\
703\end{array}$ & $\begin{array}{l}2.64 \\
97^{*}\end{array}$ & $\begin{array}{l}0.12 \\
410\end{array}$ & $\begin{array}{c}2.486 \\
21^{*}\end{array}$ & $\begin{array}{l}.31 \\
935\end{array}$ & $\begin{array}{c}13.513 \\
75^{*}\end{array}$ \\
\hline 11 & $\begin{array}{l}0.10 \\
992\end{array}$ & $\begin{array}{c}2.628 \\
83^{*}\end{array}$ & $\begin{array}{l}2.92 \\
349\end{array}$ & $\begin{array}{c}10.788 \\
99^{*}\end{array}$ & $\begin{array}{l}0.05 \\
862\end{array}$ & $\begin{array}{c}1.382 \\
09\end{array}$ & $\begin{array}{c}0.91 \\
565\end{array}$ & $94 *$ & $\begin{array}{l}0.14 \\
920\end{array}$ & $\begin{array}{c}3.407 \\
23^{*}\end{array}$ & $\begin{array}{l}400 \\
55\end{array}$ & $\begin{array}{c}15.745 \\
62 *\end{array}$ \\
\hline 12 & $\begin{array}{c}0.04 \\
531\end{array}$ & $\begin{array}{c}1.123 \\
42\end{array}$ & $\begin{array}{l}2.96 \\
880\end{array}$ & $\begin{array}{c}11.226 \\
33^{*}\end{array}$ & $\begin{array}{l}0.04 \\
055 \\
\end{array}$ & $\begin{array}{c}0.974 \\
46 \\
\end{array}$ & $\begin{array}{l}87 \\
10\end{array}$ & 3.20 & $\begin{array}{l}0.07 \\
192\end{array}$ & $\begin{array}{c}1.717 \\
37\end{array}$ & $\begin{array}{l}4.54 \\
047\end{array}$ & $\begin{array}{c}16.534 \\
59^{*}\end{array}$ \\
\hline 13 & $\begin{array}{l}0.06 \\
875\end{array}$ & $\begin{array}{c}1.484 \\
27\end{array}$ & $\begin{array}{l}3.03 \\
755\end{array}$ & $\begin{array}{c}9.8858 \\
4^{*}\end{array}$ & $\begin{array}{l}0.01 \\
929\end{array}$ & $\begin{array}{c}0.399 \\
06\end{array}$ & $\begin{array}{c}0.85 \\
581 \\
\end{array}$ & $\begin{array}{c}2.669 \\
46^{*} \\
\end{array}$ & $\begin{array}{l}0.10 \\
423\end{array}$ & $\begin{array}{c}2.203 \\
28^{*}\end{array}$ & $\begin{array}{l}4.64 \\
469\end{array}$ & $\begin{array}{c}14.802 \\
10^{*}\end{array}$ \\
\hline 14 & $\begin{array}{l}0.16 \\
716\end{array}$ & $\begin{array}{c}3.519 \\
36^{*}\end{array}$ & $\begin{array}{l}3.20 \\
471\end{array}$ & $\begin{array}{c}10.058 \\
11^{*}\end{array}$ & $\begin{array}{l}0.01 \\
155\end{array}$ & $\begin{array}{c}0.213 \\
11\end{array}$ & 0.86 & $\begin{array}{r}2.38 \\
52\end{array}$ & 0.19 & $\begin{array}{c}4.080 \\
82^{*}\end{array}$ & $\begin{array}{l}4.84 \\
372\end{array}$ & $\begin{array}{c}14.805 \\
29 *\end{array}$ \\
\hline 15 & $\begin{array}{c}0.19 \\
615\end{array}$ & $\begin{array}{c}3.735 \\
47^{*}\end{array}$ & $\begin{array}{l}3.40 \\
087\end{array}$ & $\begin{array}{c}9.5489 \\
8^{*}\end{array}$ & $\begin{array}{l}0.06 \\
042\end{array}$ & $\begin{array}{c}1.160 \\
11\end{array}$ & $\begin{array}{c}0.92 \\
778\end{array}$ & $59^{*}$ & $\begin{array}{l}0.20 \\
314\end{array}$ & $\begin{array}{c}4.328 \\
35^{*}\end{array}$ & 686 & $\begin{array}{c}15.855 \\
03 *\end{array}$ \\
\hline 16 & $\begin{array}{l}0.11 \\
756\end{array}$ & $\begin{array}{c}2.765 \\
77^{*}\end{array}$ & $\begin{array}{l}3.51 \\
843\end{array}$ & $\begin{array}{c}12.074 \\
34 *\end{array}$ & $\begin{array}{c}- \\
0.00 \\
709 \\
\end{array}$ & $\begin{array}{c}- \\
0.156 \\
71\end{array}$ & $\begin{array}{l}0.92 \\
069\end{array}$ & $\begin{array}{c}2.967 \\
38^{*}\end{array}$ & $\begin{array}{l}0.15 \\
474\end{array}$ & $\begin{array}{c}3.532 \\
77^{*}\end{array}$ & $\begin{array}{c}5.20 \\
160\end{array}$ & $\begin{array}{c}17.322 \\
30^{*}\end{array}$ \\
\hline 17 & $\begin{array}{l}0.16 \\
519\end{array}$ & $\begin{array}{c}3.885 \\
48^{*}\end{array}$ & $\begin{array}{l}3.68 \\
361\end{array}$ & $\begin{array}{c}12.506 \\
01 *\end{array}$ & $\begin{array}{r}0.00 \\
482 \\
\end{array}$ & $\begin{array}{c}0.106 \\
34\end{array}$ & $\begin{array}{l}0.91 \\
587\end{array}$ & $04^{*}$ & $\begin{array}{l}0.20 \\
340\end{array}$ & $\begin{array}{c}4.665 \\
11^{*}\end{array}$ & $\begin{array}{c}5.40 \\
500\end{array}$ & $\begin{array}{c}17.893 \\
01^{*}\end{array}$ \\
\hline 18 & $\begin{array}{c}0.23 \\
798\end{array}$ & $\begin{array}{c}5.288 \\
83^{*}\end{array}$ & $\begin{array}{c}3.92 \\
159\end{array}$ & $\begin{array}{c}12.450 \\
64^{*}\end{array}$ & $\begin{array}{l}0.04 \\
206\end{array}$ & $\begin{array}{c}0.891 \\
82\end{array}$ & $\begin{array}{l}0.95 \\
793\end{array}$ & $\begin{array}{r}2.901 \\
46^{*}\end{array}$ & $\begin{array}{l}0.27 \\
657\end{array}$ & $\begin{array}{c}6.115 \\
29^{*}\end{array}$ & & $\begin{array}{c}17.946 \\
53^{*}\end{array}$ \\
\hline 19 & $\begin{array}{c}0.23 \\
455\end{array}$ & $\begin{array}{c}4.558 \\
23^{*}\end{array}$ & 614 & $51^{*}$ & 889 & 86 & 682 & $57^{*}$ & $\begin{array}{l}0.26 \\
040\end{array}$ & $\begin{array}{c}5.322 \\
47^{*}\end{array}$ & $\begin{array}{l}5.94 \\
197\end{array}$ & $\begin{array}{c}17.176 \\
03^{*}\end{array}$ \\
\hline 20 & $\begin{array}{l}0.11 \\
275\end{array}$ & $\begin{array}{c}2.613 \\
85^{*}\end{array}$ & $\begin{array}{l}4.26 \\
889\end{array}$ & $\begin{array}{c}13.857 \\
66^{*}\end{array}$ & $\begin{array}{c}0.01 \\
861\end{array}$ & $\begin{array}{c}0.420 \\
90\end{array}$ & $\begin{array}{l}1.04 \\
543\end{array}$ & $\begin{array}{r}3.311 \\
66^{*}\end{array}$ & $\begin{array}{l}0.14 \\
424\end{array}$ & $\begin{array}{c}3.201 \\
25^{*}\end{array}$ & $\begin{array}{l}6.08 \\
621\end{array}$ & $\begin{array}{c}18.914 \\
32 *\end{array}$ \\
\hline 21 & $\begin{array}{l}0.08 \\
719\end{array}$ & $\begin{array}{c}2.077 \\
91^{*}\end{array}$ & $\begin{array}{c}4.35 \\
609\end{array}$ & $\begin{array}{c}14.395 \\
69^{*}\end{array}$ & $\begin{array}{l}0.00 \\
356\end{array}$ & $\begin{array}{c}0.084 \\
45\end{array}$ & $\begin{array}{l}1.04 \\
899\end{array}$ & $00^{*}$ & $\begin{array}{l}0.12 \\
742\end{array}$ & $\begin{array}{c}2.941 \\
96^{*}\end{array}$ & $\begin{array}{l}6.21 \\
363\end{array}$ & $\begin{array}{c}19.895 \\
25^{*}\end{array}$ \\
\hline 22 & $\begin{array}{c}0.15 \\
148\end{array}$ & $\begin{array}{c}3.632 \\
45^{*}\end{array}$ & $\begin{array}{l}4.50 \\
757\end{array}$ & $\begin{array}{c}14.847 \\
50^{*}\end{array}$ & $\begin{array}{c}0.04 \\
007\end{array}$ & $\begin{array}{c}0.900 \\
36\end{array}$ & $\begin{array}{l}1.08 \\
906\end{array}$ & $\begin{array}{c}3.361 \\
50^{*}\end{array}$ & $\begin{array}{l}0.19 \\
341\end{array}$ & $\begin{array}{c}4.512 \\
10^{*}\end{array}$ & $\begin{array}{c}6.40 \\
704\end{array}$ & $\begin{array}{c}20.531 \\
19^{*}\end{array}$ \\
\hline 23 & $\begin{array}{c}0.12 \\
950\end{array}$ & $\begin{array}{c}3.354 \\
62^{*}\end{array}$ & $\begin{array}{l}4.63 \\
707\end{array}$ & $\begin{array}{c}16.346 \\
33^{*}\end{array}$ & $\begin{array}{l}0.00 \\
733 \\
\end{array}$ & $\begin{array}{c}0.176 \\
06\end{array}$ & $\begin{array}{l}1.08 \\
173\end{array}$ & $\begin{array}{c}3.534 \\
62 *\end{array}$ & $\begin{array}{l}0.16 \\
799\end{array}$ & $\begin{array}{c}4.268 \\
38^{*}\end{array}$ & $\begin{array}{l}6.57 \\
503\end{array}$ & $\begin{array}{c}22.734 \\
35^{*}\end{array}$ \\
\hline 24 & $\begin{array}{l}0.10 \\
576\end{array}$ & $\begin{array}{c}2.532 \\
34^{*}\end{array}$ & $\begin{array}{l}4.74 \\
283\end{array}$ & $\begin{array}{c}15.312 \\
51^{*}\end{array}$ & $\begin{array}{l}0.03 \\
876\end{array}$ & $\begin{array}{c}0.870 \\
87\end{array}$ & $\begin{array}{l}1.12 \\
048\end{array}$ & $\begin{array}{c}3.395 \\
07^{*}\end{array}$ & $\begin{array}{l}0.14 \\
783\end{array}$ & $\begin{array}{c}3.415 \\
73^{*}\end{array}$ & $\begin{array}{l}6.72 \\
286\end{array}$ & $\begin{array}{c}20.945 \\
90^{*}\end{array}$ \\
\hline
\end{tabular}




\begin{tabular}{|c|c|c|c|c|c|c|c|c|c|c|c|c|}
\hline 25 & $\begin{array}{l}0.12 \\
647\end{array}$ & $\begin{array}{c}2.703 \\
22^{*}\end{array}$ & $\begin{array}{l}4.86 \\
930\end{array}$ & $\begin{array}{c}13.908 \\
47 *\end{array}$ & $\begin{array}{l}0.05 \\
159\end{array}$ & $\begin{array}{c}1.039 \\
11\end{array}$ & $\begin{array}{l}1.17 \\
207\end{array}$ & $\begin{array}{c}3.154 \\
79^{*}\end{array}$ & $\begin{array}{l}0.16 \\
485\end{array}$ & $\begin{array}{c}3.479 \\
23^{*}\end{array}$ & $\begin{array}{l}6.88 \\
771\end{array}$ & $\begin{array}{c}19.425 \\
43^{*}\end{array}$ \\
\hline 26 & 146 & $\begin{array}{c}1.038 \\
66\end{array}$ & 076 & $67^{*}$ & $\begin{array}{l}0.03 \\
767\end{array}$ & 36 & $\begin{array}{l}1.20 \\
974\end{array}$ & $51^{*}$ & 380 & $47^{*}$ & 151 & $\begin{array}{c}22.285 \\
45^{*}\end{array}$ \\
\hline 27 & $\begin{array}{l}0.00 \\
605\end{array}$ & $\begin{array}{c}0.154 \\
72\end{array}$ & $\begin{array}{c}4.90 \\
471\end{array}$ & $\begin{array}{r}16.4 \\
45\end{array}$ & $\begin{array}{l}0.00 \\
729\end{array}$ & $\begin{array}{l}.183 \\
59\end{array}$ & $\begin{array}{l}1.21 \\
704\end{array}$ & $\begin{array}{c}4.022 \\
48^{*}\end{array}$ & $\begin{array}{c}0.03 \\
154\end{array}$ & $\begin{array}{c}0.798 \\
61\end{array}$ & $\begin{array}{l}7.00 \\
304\end{array}$ & $\begin{array}{c}23.286 \\
80^{*}\end{array}$ \\
\hline 28 & $\begin{array}{l}0.02 \\
653 \\
\end{array}$ & $\begin{array}{c}0.637 \\
42\end{array}$ & $\begin{array}{l}4.87 \\
818\end{array}$ & $\begin{array}{c}15.258 \\
20^{*}\end{array}$ & $\begin{array}{l}0.01 \\
580\end{array}$ & $\begin{array}{c}0.366 \\
54 \\
\end{array}$ & $\begin{array}{l}1.20 \\
123\end{array}$ & $\begin{array}{c}3.627 \\
35^{*}\end{array}$ & $\begin{array}{c}0.00 \\
563\end{array}$ & $\begin{array}{c}0.132 \\
70\end{array}$ & $\begin{array}{l}7.00 \\
867\end{array}$ & $\begin{array}{c}21.503 \\
21^{*}\end{array}$ \\
\hline 29 & $\begin{array}{l}0.10 \\
687\end{array}$ & $\begin{array}{c}2.875 \\
50^{*}\end{array}$ & $\begin{array}{c}4.98 \\
504\end{array}$ & $\begin{array}{c}17.316 \\
46^{*}\end{array}$ & $\begin{array}{c}0.03 \\
851\end{array}$ & $\begin{array}{c}0.986 \\
51\end{array}$ & $\begin{array}{l}1.23 \\
974\end{array}$ & $40^{*}$ & 932 & $\begin{array}{c}3.543 \\
24 *\end{array}$ & 800 & $\begin{array}{c}23.468 \\
57^{*}\end{array}$ \\
\hline 3 & $\begin{array}{c}\overline{-} \\
0.00 \\
027\end{array}$ & $\begin{array}{c}-\overline{-} \\
0.007 \\
72\end{array}$ & $\begin{array}{c}4.98 \\
477\end{array}$ & $\begin{array}{c}18.155 \\
63 *\end{array}$ & $\begin{array}{l}0.00 \\
885\end{array}$ & $\begin{array}{c}0.237 \\
61\end{array}$ & $\begin{array}{l}1.24 \\
859\end{array}$ & $\begin{array}{c}4.294 \\
33^{*}\end{array}$ & $\begin{array}{l}0.04 \\
473\end{array}$ & $\begin{array}{c}1.209 \\
89\end{array}$ & $\begin{array}{l}7.19 \\
273\end{array}$ & $\begin{array}{c}24.909 \\
93^{*}\end{array}$ \\
\hline
\end{tabular}

Note: * Indicates Statistically Significant at 5\% Level of Significance

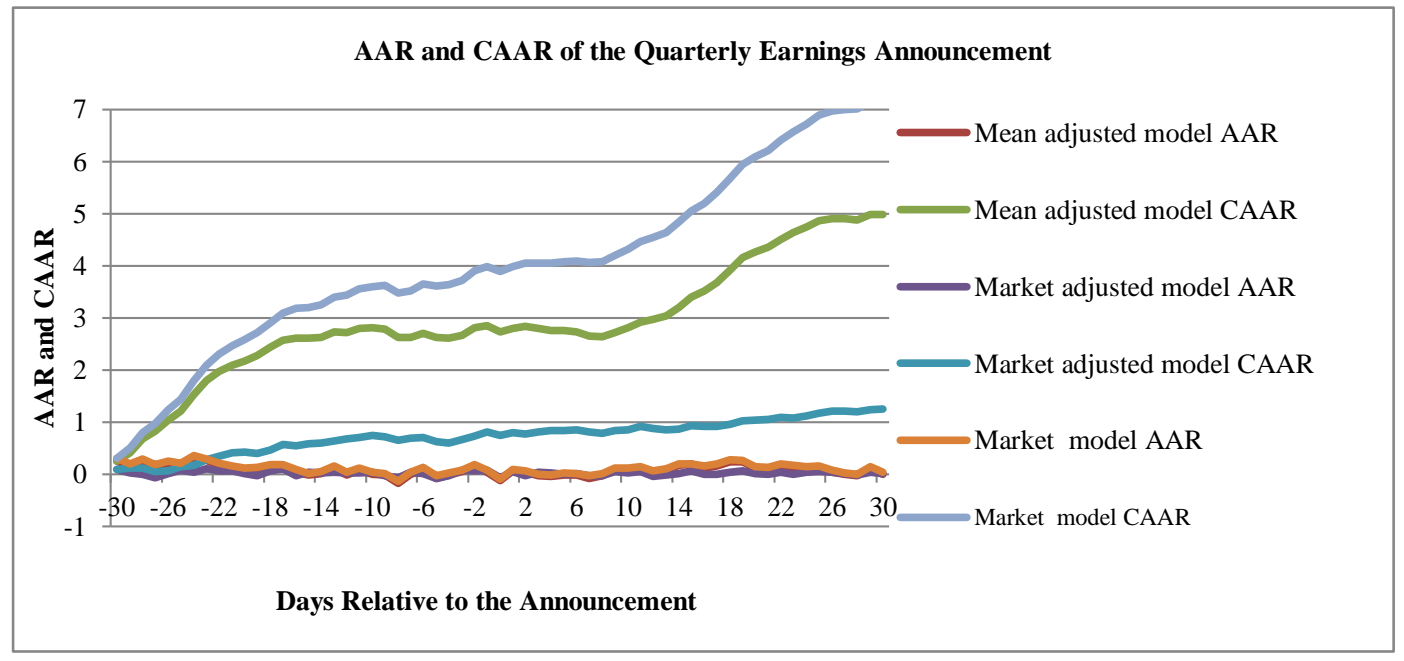

Figure 1 AARs and CAARs Trends of Three Models over the 61-Day Event Window of Full Sample Earnings Announcement of September 2012 Quarter

Table 2 and Figure 2 presents the results of good news earnings announcement. The results are presented for 61 days of event window. The results of mean adjusted model and market model exhibit similar results. The AARs are positive and significant for more than 24 days in the window period for both the models. In the case of market adjusted model, the AARs are positively insignificant for majority of the days in the event window. In particular, on the day of announcement (day 0) the AAR is positive and insignificant and on day 1 , AAR is positive and significant for all the three models. This shows that investors reacted positively on day 1 of the earnings announcement news. The results appear to be a small positive trend in event window leading up to positive returns.

To get the robust results CAAR value was calculated during the event window of 61 days. The CAAR values are found to be statistically significant for all the days in the event window for mean adjusted model and market model. Further, the CAAR 
values are increasing continuously during the window period. This phenomenon indicates that good news earnings announcement yields significant positive abnormal returns when they buy and hold during the earnings announcement. Overall, the test results indicate that AARs are close to zero and CAARs are not close to zero which generates abnormal return to the investor if they use buy and hold strategy during the earnings announcement.

Table 2 AAR and CAAR Values of Good News Earnings Announcements

\begin{tabular}{|c|c|c|c|c|c|c|c|c|c|c|c|c|}
\hline \multirow[t]{2}{*}{ Days } & \multicolumn{4}{|c|}{ Mean adjusted model } & \multicolumn{4}{|c|}{ Market adjusted model } & \multicolumn{4}{|c|}{ Market model } \\
\hline & AAR & t value & CAAR & t value & AAR & t value & CAAR & t value & AAAR & t value & CAAR & t value \\
\hline-30 & $\begin{array}{c}0.209 \\
51\end{array}$ & $\begin{array}{c}3.7965 \\
6^{*}\end{array}$ & $\begin{array}{c}0.2095 \\
1\end{array}$ & $\begin{array}{c}3.79656 \\
*\end{array}$ & 0.05505 & 0.88259 & $\begin{array}{c}0.0550 \\
5\end{array}$ & 0.88259 & $\begin{array}{c}0.2658 \\
5\end{array}$ & $\begin{array}{c}4.71016 \\
*\end{array}$ & $\begin{array}{c}0.2658 \\
5\end{array}$ & $\begin{array}{c}4.71016 \\
*\end{array}$ \\
\hline-29 & $\begin{array}{c}0.143 \\
20\end{array}$ & $\begin{array}{c}2.4366 \\
1 *\end{array}$ & $\begin{array}{c}0.3527 \\
0\end{array}$ & $\begin{array}{c}4.24371 \\
*\end{array}$ & 0.02858 & 0.44910 & $\begin{array}{c}0.0836 \\
3\end{array}$ & 0.92923 & $\begin{array}{c}0.1980 \\
7\end{array}$ & $\begin{array}{c}3.34828 \\
*\end{array}$ & $\begin{array}{c}0.4639 \\
2\end{array} \mid$ & $\begin{array}{c}5.54532 \\
*\end{array}$ \\
\hline-28 & $\begin{array}{c}0.111 \\
40\end{array}$ & $\begin{array}{c}1.9603 \\
6\end{array}$ & $\begin{array}{c}0.4641 \\
0\end{array}$ & $\begin{array}{c}4.71515 \\
*\end{array}$ & $\begin{array}{c}- \\
0.16526\end{array}$ & $\begin{array}{c}- \\
2.50989 \\
*\end{array}$ & $\begin{array}{c}- \\
0.0816 \\
3\end{array}$ & $\begin{array}{c}- \\
0.71574\end{array}$ & $\begin{array}{c}0.1645 \\
4\end{array}$ & $\mid \begin{array}{c}2.89858 \\
*\end{array}$ & $\begin{array}{c}0.6284 \\
6\end{array}$ & $\begin{array}{c}6.39209 \\
*\end{array}$ \\
\hline-27 & $\begin{array}{c}0.099 \\
40\end{array}$ & $\begin{array}{c}1.7787 \\
4\end{array}$ & $\begin{array}{c}0.5635 \\
0\end{array}$ & $\begin{array}{c}5.04202 \\
*\end{array}$ & -0.13260 & $\begin{array}{c}- \\
2.25045 \\
*\end{array}$ & $\begin{array}{c}- \\
0.2142 \\
3\end{array}$ & $\begin{array}{c}- \\
1.81788\end{array}$ & $\begin{array}{c}0.1516 \\
5\end{array}$ & $\mid \begin{array}{c}2.73874 \\
*\end{array}$ & $\begin{array}{c}0.7801 \\
1\end{array}$ & $\begin{array}{c}7.04417 \\
*\end{array}$ \\
\hline-26 & $\begin{array}{c}0.250 \\
34\end{array}$ & $\begin{array}{c}3.5518 \\
9^{*}\end{array}$ & $\begin{array}{c}0.8138 \\
4\end{array}$ & $\begin{array}{c}5.16390 \\
*\end{array}$ & 0.05319 & 0.74308 & $\begin{array}{c}- \\
0.1610 \\
4\end{array}$ & $\begin{array}{c}- \\
1.00619\end{array}$ & $\begin{array}{c}0.2962 \\
9\end{array}$ & $\begin{array}{c}4.55875 \\
*\end{array}$ & $\begin{array}{c}1.0764 \\
0\end{array}$ & $\begin{array}{c}7.40652 \\
*\end{array}$ \\
\hline-25 & $\begin{array}{c}0.277 \\
75\end{array}$ & $\begin{array}{c}4.6866 \\
4 *\end{array}$ & $\begin{array}{c}1.0916 \\
0\end{array}$ & $\begin{array}{c}7.51949 \\
*\end{array}$ & 0.21144 & $\begin{array}{c}3.40517 \\
*\end{array}$ & $\begin{array}{c}0.0504 \\
0\end{array}$ & 0.33136 & $\begin{array}{c}0.3297 \\
1\end{array}$ & $\begin{array}{c}5.56178 \\
*\end{array}$ & $\begin{array}{c}1.4061 \\
1\end{array}$ & $\begin{array}{c}9.68338 \\
*\end{array}$ \\
\hline-24 & $\begin{array}{c}0.278 \\
09\end{array}$ & $\begin{array}{c}4.1697 \\
8^{*}\end{array}$ & $\begin{array}{c}1.3696 \\
8\end{array}$ & $\begin{array}{c}7.76257 \\
*\end{array}$ & -0.01235 & $\begin{array}{c}- \\
0.16130\end{array}$ & $\begin{array}{c}0.0380 \\
5\end{array}$ & 0.18783 & $\begin{array}{c}0.3297 \\
2\end{array}$ & $\begin{array}{c}4.89788 \\
*\end{array}$ & $\begin{array}{c}1.7358 \\
3\end{array}$ & $\begin{array}{c}9.74599 \\
*\end{array}$ \\
\hline-23 & $\begin{array}{c}0.247 \\
32\end{array}$ & $\begin{array}{c}4.1093 \\
3^{*}\end{array}$ & $\begin{array}{c}1.6170 \\
1\end{array}$ & $\begin{array}{c}9.49891 \\
*\end{array}$ & 0.09512 & 1.46503 & $\begin{array}{c}0.1331 \\
7\end{array}$ & 0.72516 & $\begin{array}{c}0.2976 \\
9\end{array}$ & $\begin{array}{c}5.04015 \\
*\end{array}$ & $\begin{array}{c}2.0335 \\
2\end{array}$ & $\begin{array}{c}12.1725 \\
3^{*}\end{array}$ \\
\hline-22 & $\begin{array}{c}0.194 \\
42\end{array}$ & $\begin{array}{c}3.2349 \\
0^{*}\end{array}$ & $\begin{array}{c}1.8114 \\
3\end{array}$ & $\begin{array}{c}10.0464 \\
0 *\end{array}$ & 0.06729 & 1.11640 & $\begin{array}{c}0.2004 \\
6\end{array}$ & 1.10867 & $\begin{array}{c}0.2460 \\
4\end{array}$ & $\begin{array}{c}4.09051 \\
*\end{array}$ & $\begin{array}{c}2.2795 \\
6\end{array}$ & $\begin{array}{c}12.6330 \\
0^{*}\end{array}$ \\
\hline-21 & $\begin{array}{c}0.094 \\
78\end{array}$ & $\begin{array}{c}1.6526 \\
9\end{array}$ & $\begin{array}{c}1.9062 \\
1\end{array}$ & $\begin{array}{c}10.5115 \\
5^{*}\end{array}$ & 0.05843 & 0.93959 & $\begin{array}{c}0.2588 \\
9\end{array}$ & 1.31651 & $\begin{array}{c}0.1473 \\
1\end{array}$ & $\begin{array}{c}2.46878 \\
*\end{array}$ & $\begin{array}{c}2.4268 \\
7\end{array}$ & $\begin{array}{c}12.8613 \\
5^{*}\end{array}$ \\
\hline-20 & $\begin{array}{c}- \\
0.003 \\
13\end{array}$ & $\begin{array}{c}- \\
0.0520 \\
3\end{array}$ & $\begin{array}{c}1.9030 \\
7\end{array}$ & $\begin{array}{c}9.52405 \\
*\end{array}$ & -0.08577 & $\mid \begin{array}{c}- \\
1.40428\end{array}$ & $\begin{array}{c}0.1731 \\
2\end{array}$ & 0.85465 & $\begin{array}{c}0.0525 \\
3\end{array}$ & 0.85942 & $\begin{array}{c}2.4794 \\
0\end{array}$ & $\begin{array}{c}12.2308 \\
7 *\end{array}$ \\
\hline-19 & $\begin{array}{c}0.116 \\
48\end{array}$ & $\begin{array}{c}1.6159 \\
0\end{array}$ & $\begin{array}{c}2.0195 \\
5\end{array}$ & $\begin{array}{c}8.08766 \\
*\end{array}$ & -0.00545 & $\begin{array}{c}- \\
0.07498\end{array}$ & $\begin{array}{c}0.1676 \\
7\end{array}$ & 0.66563 & $\begin{array}{c}0.1666 \\
9\end{array}$ & $\begin{array}{c}2.26668 \\
*\end{array}$ & $\begin{array}{c}2.6460 \\
9\end{array}$ & $\begin{array}{c}10.3872 \\
1 *\end{array}$ \\
\hline-18 & $\begin{array}{c}0.225 \\
36\end{array}$ & $\begin{array}{c}3.6680 \\
6^{*}\end{array}$ & $\begin{array}{c}2.2449 \\
2\end{array}$ & $\begin{array}{c}10.1340 \\
7 *\end{array}$ & 0.16773 & $\begin{array}{c}2.75012 \\
*\end{array}$ & $\begin{array}{c}0.3354 \\
0\end{array}$ & 1.52519 & $\begin{array}{c}0.2764 \\
1\end{array}$ & $\begin{array}{c}4.46106 \\
*\end{array}$ & $\begin{array}{c}2.9225 \\
0\end{array}$ & $\begin{array}{c}13.0816 \\
7 *\end{array}$ \\
\hline-17 & $\begin{array}{c}0.169 \\
74\end{array}$ & $\begin{array}{c}3.1479 \\
3^{*}\end{array}$ & $\begin{array}{c}2.4146 \\
5\end{array}$ & $\begin{array}{c}11.9684 \\
3 *\end{array}$ & 0.14502 & $\mid \begin{array}{c}2.47422 \\
*\end{array}$ & $\begin{array}{c}0.4804 \\
2\end{array}$ & $\left|\begin{array}{c}2.19065 \\
*\end{array}\right|$ & $\begin{array}{c}0.2233 \\
6\end{array}$ & $\begin{array}{c}4.08856 \\
*\end{array}$ & $\begin{array}{c}3.1458 \\
6\end{array}$ & $\begin{array}{c}15.3902 \\
8^{*}\end{array}$ \\
\hline-16 & $\begin{array}{c}0.044 \\
16\end{array}$ & $\begin{array}{c}0.8295 \\
1\end{array}$ & $\begin{array}{c}2.4588 \\
1\end{array}$ & $\begin{array}{c}11.9264 \\
7 *\end{array}$ & -0.02044 & $\begin{array}{c}- \\
0.37816\end{array}$ & $\begin{array}{c}0.4599 \\
8\end{array}$ & $\left|\begin{array}{c}2.19781 \\
*\end{array}\right|$ & $\begin{array}{c}0.0973 \\
5\end{array}$ & 1.78019 & $\begin{array}{c}3.2432 \\
1\end{array}$ & $\begin{array}{c}15.3124 \\
9 *\end{array}$ \\
\hline
\end{tabular}




\begin{tabular}{|c|c|c|c|c|c|c|c|c|c|c|c|c|}
\hline-15 & $\begin{array}{c}- \\
0.066 \\
42 \\
\end{array}$ & $\begin{array}{c}- \\
1.1625 \\
9 \\
\end{array}$ & $\begin{array}{c}2.3923 \\
9\end{array}$ & $\begin{array}{c}10.4693 \\
8^{*}\end{array}$ & -0.00880 & $\begin{array}{c}- \\
0.15342\end{array}$ & $\begin{array}{c}0.4511 \\
8\end{array}$ & 1.96609 & \begin{tabular}{|c}
- \\
0.0147 \\
5
\end{tabular} & $\begin{array}{c}- \\
0.25685\end{array}$ & $\begin{array}{c}3.2284 \\
6\end{array}$ & $\begin{array}{c}14.0521 \\
0^{*}\end{array}$ \\
\hline-14 & $\begin{array}{c}0.052 \\
51\end{array}$ & $\begin{array}{c}1.0119 \\
3\end{array}$ & $\begin{array}{c}2.4449 \\
1\end{array}$ & $\begin{array}{c}11.4264 \\
3^{*}\end{array}$ & 0.08128 & 1.34841 & $\begin{array}{c}0.5324 \\
6\end{array}$ & $\begin{array}{c}2.14234 \\
*\end{array}$ & $\begin{array}{c}0.1075 \\
8\end{array}$ & $\begin{array}{c}2.01154 \\
*\end{array}$ & $\begin{array}{c}3.3360 \\
4\end{array}$ & $\begin{array}{c}15.1281 \\
0 *\end{array}$ \\
\hline-13 & $\begin{array}{c}0.082 \\
48\end{array}$ & $\begin{array}{c}1.5230 \\
3\end{array}$ & $\begin{array}{c}2.5273 \\
9\end{array}$ & $\begin{array}{c}10.9999 \\
6^{*}\end{array}$ & 0.01462 & 0.25245 & $\begin{array}{c}0.5470 \\
9\end{array}$ & $\begin{array}{c}2.22599 \\
*\end{array}$ & $\begin{array}{c}0.1368 \\
8\end{array}$ & $\mid \begin{array}{c}2.39367 \\
*\end{array}$ & $\begin{array}{c}3.4729 \\
2\end{array}$ & $\begin{array}{c}14.3144 \\
8 *\end{array}$ \\
\hline-12 & $\begin{array}{c}0.014 \\
30\end{array}$ & $\begin{array}{c}0.2412 \\
8\end{array}$ & $\begin{array}{c}2.5416 \\
9\end{array}$ & $\begin{array}{c}9.83730 \\
*\end{array}$ & 0.07040 & 1.18392 & $\begin{array}{c}0.6174 \\
9\end{array}$ & $\begin{array}{c}2.38240 \\
*\end{array}$ & $\begin{array}{c}0.0671 \\
4\end{array}$ & 1.09213 & $\begin{array}{c}3.5400 \\
7\end{array}$ & $\begin{array}{c}13.2103 \\
9^{*}\end{array}$ \\
\hline-11 & $\begin{array}{c}0.027 \\
91\end{array}$ & $\begin{array}{c}0.4402 \\
5\end{array}$ & $\begin{array}{c}2.5696 \\
0\end{array}$ & $\begin{array}{c}9.06402 \\
*\end{array}$ & -0.02998 & $\begin{array}{c}- \\
0.47350\end{array}$ & $\begin{array}{c}0.5875 \\
1\end{array}$ & $\begin{array}{c}2.07503 \\
*\end{array}$ & $\begin{array}{c}0.0817 \\
0\end{array}$ & 1.27554 & $\begin{array}{c}3.6217 \\
6\end{array}$ & $\begin{array}{c}12.6444 \\
2 *\end{array}$ \\
\hline-10 & $\begin{array}{c}0.003 \\
60\end{array}$ & $\begin{array}{c}0.0725 \\
9\end{array}$ & $\begin{array}{c}2.5732 \\
0\end{array}$ & $\begin{array}{c}11.3290 \\
5^{*}\end{array}$ & 0.06317 & 1.17430 & $\begin{array}{c}0.6506 \\
8\end{array}$ & $\begin{array}{c}2.63951 \\
*\end{array}$ & $\begin{array}{c}0.0586 \\
3\end{array}$ & 1.13954 & $\begin{array}{c}3.6803 \\
9\end{array}$ & $\begin{array}{c}15.6096 \\
0^{*}\end{array}$ \\
\hline-9 & $\begin{array}{c}0.028 \\
62\end{array}$ & $\begin{array}{c}0.5571 \\
0\end{array}$ & $\begin{array}{c}2.6018 \\
2\end{array}$ & $\begin{array}{c}10.7973 \\
7 *\end{array}$ & 0.00919 & 0.17709 & $\begin{array}{c}0.6598 \\
7\end{array}$ & $\begin{array}{c}2.71062 \\
*\end{array}$ & $\begin{array}{c}0.0819 \\
8\end{array}$ & 1.51966 & $\begin{array}{c}3.7623 \\
7\end{array}$ & $\begin{array}{c}14.8692 \\
9 *\end{array}$ \\
\hline-8 & $\begin{array}{c}- \\
0.279 \\
11 \\
\end{array}$ & \begin{tabular}{|c}
- \\
4.7507 \\
$3^{*}$
\end{tabular} & $\begin{array}{c}2.3227 \\
0\end{array}$ & $\begin{array}{c}8.24351 \\
*\end{array}$ & -0.16582 & $\begin{array}{c}- \\
2.63914 \\
*\end{array}$ & $\begin{array}{c}0.4940 \\
5\end{array}$ & 1.63954 & $\begin{array}{c}- \\
0.2245 \\
8\end{array}$ & $\begin{array}{c}- \\
3.65030 \\
*\end{array}$ & $\begin{array}{c}3.5377 \\
9\end{array}$ & $\begin{array}{c}11.9901 \\
3^{*}\end{array}$ \\
\hline-7 & $\begin{array}{c}0.048 \\
59\end{array}$ & $\begin{array}{c}0.8306 \\
3\end{array}$ & $\begin{array}{c}2.3713 \\
0\end{array}$ & $\begin{array}{c}8.27388 \\
*\end{array}$ & 0.10581 & 1.73099 & $\begin{array}{c}0.5998 \\
5\end{array}$ & $\begin{array}{c}2.00321 \\
*\end{array}$ & $\begin{array}{c}0.1018 \\
3\end{array}$ & 1.68773 & $\begin{array}{c}3.6396 \\
2\end{array}$ & $\begin{array}{c}12.3131 \\
1^{*}\end{array}$ \\
\hline-6 & $\begin{array}{c}0.136 \\
03\end{array}$ & $\begin{array}{c}2.7918 \\
5^{*}\end{array}$ & $\begin{array}{c}2.5073 \\
3\end{array}$ & $\begin{array}{c}10.2921 \\
9^{*}\end{array}$ & 0.06650 & 1.32187 & $\begin{array}{c}0.6663 \\
5\end{array}$ & $\begin{array}{c}2.64917 \\
*\end{array}$ & $\begin{array}{c}0.1901 \\
3\end{array}$ & $\mid \begin{array}{c}3.77314 \\
*\end{array}$ & $\begin{array}{c}3.8297 \\
6\end{array}$ & $\begin{array}{c}15.2000 \\
4^{*}\end{array}$ \\
\hline-5 & $\begin{array}{c}- \\
0.030 \\
46 \\
\end{array}$ & $\begin{array}{c}- \\
0.5418 \\
6\end{array}$ & $\begin{array}{c}2.4768 \\
6\end{array}$ & $\begin{array}{c}8.64024 \\
*\end{array}$ & -0.01249 & $\begin{array}{c}- \\
0.21117\end{array}$ & $\begin{array}{c}0.6538 \\
6\end{array}$ & $\begin{array}{c}2.16779 \\
*\end{array}$ & $\begin{array}{c}0.0217 \\
4\end{array}$ & 0.37965 & $\begin{array}{c}3.8515 \\
0\end{array}$ & $\begin{array}{c}13.1917 \\
8^{*}\end{array}$ \\
\hline-4 & $\begin{array}{c}- \\
0.000 \\
72\end{array}$ & $\begin{array}{c}- \\
0.0143 \\
4\end{array}$ & $\begin{array}{c}2.4761 \\
4\end{array}$ & $\begin{array}{c}9.52093 \\
*\end{array}$ & -0.01231 & $\begin{array}{c}- \\
0.23335\end{array}$ & $\begin{array}{c}0.6415 \\
5\end{array}$ & $\begin{array}{c}2.34058 \\
*\end{array}$ & $\begin{array}{c}0.0543 \\
4\end{array}$ & 1.02034 & $\begin{array}{c}3.9058 \\
3\end{array}$ & $\begin{array}{c}14.1152 \\
3^{*}\end{array}$ \\
\hline-3 & $\begin{array}{c}0.046 \\
90\end{array}$ & $\begin{array}{c}0.8699 \\
0\end{array}$ & $\begin{array}{c}2.5230 \\
5\end{array}$ & $\begin{array}{c}8.84321 \\
*\end{array}$ & 0.09880 & 1.69523 & $\begin{array}{c}0.7403 \\
5\end{array}$ & $\begin{array}{c}2.40057 \\
*\end{array}$ & $\begin{array}{c}0.1014 \\
5\end{array}$ & 1.79448 & $\begin{array}{c}4.0072 \\
8\end{array}$ & $\begin{array}{c}13.3951 \\
3^{*}\end{array}$ \\
\hline-2 & $\begin{array}{c}0.149 \\
63\end{array}$ & $\begin{array}{c}2.4553 \\
8^{*}\end{array}$ & $\begin{array}{c}2.6726 \\
8\end{array}$ & $\begin{array}{c}8.14408 \\
*\end{array}$ & 0.06095 & 0.93768 & $\begin{array}{c}0.8013 \\
1\end{array}$ & $\begin{array}{c}2.28909 \\
*\end{array}$ & $\begin{array}{c}0.2001 \\
5\end{array}$ & $\left|\begin{array}{c}3.20352 \\
*\end{array}\right|$ & $\begin{array}{c}4.2074 \\
4\end{array}$ & $\begin{array}{c}12.5050 \\
1 *\end{array}$ \\
\hline-1 & $\begin{array}{c}0.001 \\
45\end{array}$ & $\begin{array}{c}0.0286 \\
3\end{array}$ & $\begin{array}{c}2.6741 \\
3\end{array}$ & $\begin{array}{c}9.60838 \\
*\end{array}$ & 0.04682 & 0.88469 & $\begin{array}{c}0.8481 \\
3\end{array}$ & $\begin{array}{c}2.92592 \\
*\end{array}$ & $\begin{array}{c}0.0537 \\
8\end{array}$ & 1.03061 & $\begin{array}{c}4.2612 \\
2\end{array}$ & $\begin{array}{c}14.9090 \\
7 *\end{array}$ \\
\hline 0 & $\begin{array}{c}0.072 \\
46\end{array}$ & $\begin{array}{c}0.8662 \\
3\end{array}$ & $\begin{array}{c}2.7465 \\
9\end{array}$ & $\begin{array}{c}5.89739 \\
*\end{array}$ & 0.13743 & 1.55305 & $\begin{array}{c}0.9855 \\
5\end{array}$ & $\begin{array}{c}2.00038 \\
*\end{array}$ & $\begin{array}{c}0.1159 \\
2\end{array}$ & 1.38829 & $\begin{array}{c}4.3771 \\
3\end{array}$ & $\begin{array}{c}9.41539 \\
*\end{array}$ \\
\hline 1 & $\begin{array}{c}0.213 \\
26\end{array}$ & $\begin{array}{c}2.3647 \\
9 *\end{array}$ & $\begin{array}{c}2.9598 \\
5\end{array}$ & $\begin{array}{c}5.80207 \\
*\end{array}$ & 0.20835 & $\mid \begin{array}{c}2.25313 \\
*\end{array}$ & $\begin{array}{c}1.1939 \\
0\end{array}$ & $\begin{array}{c}2.28238 \\
*\end{array}$ & $\begin{array}{c}0.2565 \\
6\end{array}$ & $\begin{array}{c}2.81246 \\
*\end{array}$ & $\begin{array}{c}4.6336 \\
9\end{array}$ & $\begin{array}{c}8.97947 \\
*\end{array}$ \\
\hline 2 & $\begin{array}{c}0.047 \\
30\end{array}$ & $\begin{array}{c}0.8325 \\
8\end{array}$ & $\begin{array}{c}3.0071 \\
4\end{array}$ & $\begin{array}{c}9.21525 \\
*\end{array}$ & -0.01642 & $\begin{array}{c}- \\
0.26611\end{array}$ & $\begin{array}{c}1.1774 \\
8\end{array}$ & $\begin{array}{c}3.32130 \\
*\end{array}$ & $\begin{array}{c}0.0987 \\
8\end{array}$ & 1.72760 & $\begin{array}{c}4.7324 \\
7\end{array}$ & $\begin{array}{c}14.4079 \\
4 *\end{array}$ \\
\hline 3 & $\begin{array}{c}- \\
0.006 \\
36\end{array}$ & $\begin{array}{c}- \\
0.1155 \\
3 \\
\end{array}$ & $\begin{array}{c}3.0007 \\
9\end{array}$ & $\begin{array}{c}9.35543 \\
*\end{array}$ & 0.07130 & 1.26924 & $\begin{array}{c}1.2487 \\
8\end{array}$ & $\begin{array}{c}3.81218 \\
*\end{array}$ & $\begin{array}{c}0.0482 \\
4\end{array}$ & 0.86565 & $\begin{array}{c}4.7807 \\
2\end{array}$ & $\begin{array}{c}14.7117 \\
9 *\end{array}$ \\
\hline 4 & $\begin{array}{c}- \\
0.001 \\
14\end{array}$ & $\begin{array}{c}- \\
0.0209 \\
4\end{array}$ & $\begin{array}{c}2.9996 \\
4\end{array}$ & $\begin{array}{c}9.27191 \\
*\end{array}$ & 0.07323 & 1.30669 & $\begin{array}{c}1.3220 \\
1\end{array}$ & $\begin{array}{c}3.98739 \\
*\end{array}$ & $\begin{array}{c}0.0517 \\
2\end{array}$ & 0.92805 & $\begin{array}{c}4.8324 \\
3\end{array}$ & $\begin{array}{c}14.6582 \\
4 *\end{array}$ \\
\hline
\end{tabular}




\begin{tabular}{|c|c|c|c|c|c|c|c|c|c|c|c|c|}
\hline 5 & $\begin{array}{c}0.020 \\
89 \\
\end{array}$ & \begin{tabular}{|c|}
- \\
0.3803 \\
1 \\
\end{tabular} & $\left|\begin{array}{c}2.9787 \\
5\end{array}\right|$ & $\begin{array}{c}9.03726 \\
*\end{array}$ & -0.00999 & $\begin{array}{c}- \\
0.17777\end{array}$ & $\begin{array}{c}1.3120 \\
2\end{array}$ & $\left|\begin{array}{c}3.88972 \\
*\end{array}\right|$ & $\left|\begin{array}{c}0.0275 \\
6\end{array}\right|$ & 0.48344 & $\begin{array}{c}4.8600 \\
0\end{array}$ & $\begin{array}{c}14.2060 \\
5^{*}\end{array}$ \\
\hline 6 & $\begin{array}{c}0.039 \\
92\end{array}$ & $\begin{array}{c}0.7498 \\
6\end{array}$ & $\mid \begin{array}{c}3.0186 \\
8\end{array}$ & $\begin{array}{c}9.32096 \\
*\end{array}$ & 0.06941 & 1.23161 & $\begin{array}{c}1.3814 \\
3\end{array}$ & $\begin{array}{c}4.02961 \\
*\end{array}$ & $\begin{array}{c}0.0935 \\
6\end{array}$ & 1.65879 & $\begin{array}{c}4.9535 \\
5\end{array}$ & $\begin{array}{c}14.4388 \\
0^{*}\end{array}$ \\
\hline 7 & $\begin{array}{c}- \\
0.018 \\
06\end{array}$ & $\begin{array}{c}- \\
0.3662 \\
7\end{array}$ & $\begin{array}{c}3.0006 \\
2\end{array}$ & $\begin{array}{c}9.87217 \\
*\end{array}$ & 0.05409 & 1.05978 & $\begin{array}{c}1.4355 \\
2\end{array}$ & $\begin{array}{c}4.56253 \\
*\end{array}$ & $\left|\begin{array}{c}0.0326 \\
7\end{array}\right|$ & 0.65114 & $\begin{array}{c}4.9862 \\
2\end{array}$ & $\begin{array}{c}16.1212 \\
5^{*}\end{array}$ \\
\hline 8 & $\begin{array}{c}0.044 \\
45\end{array}$ & $\begin{array}{c}0.8251 \\
4\end{array}$ & $\begin{array}{c}3.0450 \\
7\end{array}$ & $\begin{array}{c}9.05103 \\
*\end{array}$ & 0.05613 & 1.06046 & $\begin{array}{c}1.4916 \\
6\end{array}$ & $\begin{array}{c}4.51234 \\
*\end{array}$ & $\begin{array}{c}0.0950 \\
7\end{array}$ & 1.73273 & $\begin{array}{c}5.0812 \\
9\end{array}$ & $\begin{array}{c}14.8300 \\
7 *\end{array}$ \\
\hline 9 & $\begin{array}{c}0.084 \\
89\end{array}$ & $\begin{array}{c}1.3425 \\
3\end{array}$ & $\begin{array}{c}3.1299 \\
6\end{array}$ & $\begin{array}{c}7.82662 \\
*\end{array}$ & 0.05931 & 0.91270 & $\begin{array}{c}1.5509 \\
7\end{array}$ & $\begin{array}{c}3.77374 \\
*\end{array}$ & $\begin{array}{c}0.1371 \\
9\end{array}$ & $\begin{array}{c}2.15092 \\
*\end{array}$ & $\begin{array}{c}5.2184 \\
8\end{array}$ & $\begin{array}{c}12.9365 \\
1^{*}\end{array}$ \\
\hline 10 & $\begin{array}{c}0.050 \\
24\end{array}$ & $\begin{array}{c}0.8210 \\
7\end{array}$ & $\mid \begin{array}{c}3.1802 \\
0\end{array}$ & $\begin{array}{c}8.11712 \\
*\end{array}$ & -0.00902 & $\begin{array}{c}- \\
0.14442\end{array}$ & $\begin{array}{c}1.5419 \\
5\end{array}$ & $\begin{array}{c}3.85463 \\
*\end{array}$ & $\begin{array}{c}0.1014 \\
7\end{array}$ & 1.62585 & $\begin{array}{c}5.3199 \\
5\end{array}$ & $\begin{array}{c}13.3121 \\
9^{*}\end{array}$ \\
\hline 11 & $\begin{array}{c}0.140 \\
45\end{array}$ & $\begin{array}{c}2.2494 \\
2^{*}\end{array}$ & $\begin{array}{c}3.3206 \\
5\end{array}$ & $\begin{array}{c}8.20614 \\
*\end{array}$ & 0.10802 & 1.71215 & $\begin{array}{c}1.6499 \\
7\end{array}$ & $\begin{array}{c}4.03541 \\
*\end{array}$ & $\begin{array}{c}0.1928 \\
5\end{array}$ & $\begin{array}{c}3.02480 \\
*\end{array}$ & $\begin{array}{c}5.5128 \\
1\end{array}$ & $\begin{array}{c}13.3419 \\
6^{*}\end{array}$ \\
\hline 12 & $\begin{array}{c}0.056 \\
81\end{array}$ & $\begin{array}{c}1.0271 \\
8\end{array}$ & $\begin{array}{c}3.3774 \\
6\end{array}$ & $\begin{array}{c}9.31249 \\
*\end{array}$ & $-0.00880 \mid$ & $\mid \begin{array}{c}- \\
0.15498\end{array}$ & $\begin{array}{c}1.6411 \\
6\end{array}$ & $\begin{array}{c}4.40587 \\
*\end{array}$ & $\begin{array}{c}0.1105 \\
8\end{array}$ & $\begin{array}{c}1.98139 \\
*\end{array}$ & $\begin{array}{c}5.6233 \\
9\end{array}$ & $\begin{array}{c}15.3653 \\
6^{*}\end{array}$ \\
\hline 13 & $\begin{array}{c}0.073 \\
02\end{array}$ & $\begin{array}{c}1.1417 \\
0\end{array}$ & $\begin{array}{c}3.4504 \\
8\end{array}$ & $\begin{array}{c}8.13370 \\
*\end{array}$ & 0.00163 & 0.02359 & $\begin{array}{c}1.6427 \\
9\end{array}$ & $\begin{array}{c}3.58043 \\
*\end{array}$ & $\begin{array}{c}0.1264 \\
1\end{array}$ & 1.94664 & $\begin{array}{c}5.7498 \\
0\end{array}$ & $\begin{array}{c}13.3484 \\
4^{*}\end{array}$ \\
\hline 14 & $\begin{array}{c}0.118 \\
79\end{array}$ & $\begin{array}{c}1.8678 \\
3\end{array}$ & $\begin{array}{c}3.5692 \\
6\end{array}$ & $\begin{array}{c}8.36657 \\
*\end{array}$ & -0.03392 & $\mid \begin{array}{c}- \\
0.46187\end{array}$ & $\begin{array}{c}1.6088 \\
7\end{array}$ & $\begin{array}{c}3.26548 \\
*\end{array}$ & $\begin{array}{c}0.1721 \\
3\end{array}$ & $\begin{array}{c}2.68574 \\
*\end{array}$ & $\begin{array}{c}5.9219 \\
3\end{array}$ & $\begin{array}{c}13.7742 \\
1^{*}\end{array}$ \\
\hline 15 & $\begin{array}{c}0.199 \\
87\end{array}$ & $\begin{array}{c}2.4229 \\
7 *\end{array}$ & $\begin{array}{c}3.7691 \\
3\end{array}$ & $\begin{array}{c}6.73710 \\
*\end{array}$ & 0.05834 & 0.73907 & $\begin{array}{c}1.6672 \\
1\end{array}$ & $\begin{array}{c}3.11420 \\
*\end{array}$ & $\begin{array}{c}0.2163 \\
4\end{array}$ & $\begin{array}{c}3.33974 \\
*\end{array}$ & $\begin{array}{c}6.1382 \\
7\end{array}$ & $\begin{array}{c}13.9712 \\
8^{*}\end{array}$ \\
\hline 16 & $\begin{array}{c}0.137 \\
05\end{array}$ & $\begin{array}{c}2.4387 \\
6^{*}\end{array}$ & $\mid \begin{array}{c}3.9061 \\
8\end{array}$ & $\begin{array}{c}10.1387 \\
0^{*}\end{array}$ & 0.01454 & 0.25063 & $\begin{array}{c}1.6817 \\
5\end{array}$ & $\begin{array}{c}4.22741 \\
*\end{array}$ & $\begin{array}{c}0.1903 \\
5\end{array} \mid$ & $\begin{array}{c}3.37769 \\
*\end{array}$ & $\begin{array}{c}6.3286 \\
2\end{array}$ & $\begin{array}{c}16.3807 \\
2^{*}\end{array}$ \\
\hline 17 & $\begin{array}{c}0.189 \\
60\end{array}$ & $\begin{array}{c}2.9464 \\
2^{*}\end{array}$ & $\begin{array}{c}4.0957 \\
8\end{array}$ & $\begin{array}{c}9.18693 \\
*\end{array}$ & 0.02172 & 0.31649 & $\begin{array}{c}1.7034 \\
8\end{array}$ & $\begin{array}{c}3.58223 \\
*\end{array}$ & $\begin{array}{c}0.2397 \\
4\end{array}$ & $\begin{array}{c}3.71469 \\
*\end{array}$ & $\begin{array}{c}6.5683 \\
6\end{array}$ & $\begin{array}{c}14.6901 \\
2^{*}\end{array}$ \\
\hline 18 & $\begin{array}{c}0.288 \\
26\end{array}$ & $\begin{array}{c}4.3423 \\
6^{*}\end{array}$ & $\begin{array}{c}4.3840 \\
5\end{array}$ & $\begin{array}{c}9.43437 \\
*\end{array}$ & 0.10214 & 1.50055 & $\begin{array}{c}1.8056 \\
1\end{array}$ & $\begin{array}{c}3.78962 \\
*\end{array}$ & $\begin{array}{c}0.3393 \\
1\end{array}$ & $\begin{array}{c}5.11345 \\
*\end{array}$ & $\begin{array}{c}6.9076 \\
6\end{array}$ & $\begin{array}{c}14.8714 \\
8^{*}\end{array}$ \\
\hline 19 & $\begin{array}{c}0.175 \\
86\end{array}$ & $\begin{array}{c}2.2526 \\
4 *\end{array}$ & $\begin{array}{c}4.5599 \\
0\end{array}$ & $\begin{array}{c}8.26050 \\
*\end{array}$ & -0.00296 & $\mid \begin{array}{c}- \\
0.03921\end{array}$ & $\begin{array}{c}1.8026 \\
5\end{array}$ & $\begin{array}{c}3.37514 \\
*\end{array}$ & $\begin{array}{c}0.2142 \\
9\end{array}$ & $\begin{array}{c}2.94964 \\
*\end{array}$ & $\begin{array}{c}7.1219 \\
5\end{array}$ & $\begin{array}{c}13.8636 \\
7 *\end{array}$ \\
\hline 20 & $\begin{array}{c}0.054 \\
51\end{array}$ & $\begin{array}{c}0.8419 \\
7\end{array}$ & $\begin{array}{c}4.6144 \\
2\end{array}$ & $\begin{array}{c}9.97970 \\
*\end{array}$ & -0.03862 & $\begin{array}{c}- \\
0.57768\end{array}$ & $\begin{array}{c}1.7640 \\
3\end{array}$ & $\begin{array}{c}3.69486 \\
*\end{array}$ & $\begin{array}{c}0.1055 \\
9\end{array}$ & 1.56376 & $\begin{array}{c}7.2275 \\
5\end{array}$ & $\begin{array}{c}14.9879 \\
5^{*}\end{array}$ \\
\hline 21 & $\begin{array}{c}0.064 \\
30\end{array}$ & $\begin{array}{c}1.1352 \\
6\end{array}$ & $\begin{array}{c}4.6787 \\
1\end{array}$ & $\begin{array}{c}11.4556 \\
2^{*}\end{array}$ & -0.01454 & $\mid \begin{array}{c}- \\
0.25594\end{array}$ & $\begin{array}{c}1.7494 \\
9\end{array}$ & $\begin{array}{c}4.27066 \\
*\end{array}$ & $\begin{array}{c}0.1165 \\
0\end{array}$ & 1.96472 & $\begin{array}{c}7.3440 \\
5\end{array}$ & $\begin{array}{c}17.1749 \\
3^{*}\end{array}$ \\
\hline 22 & $\begin{array}{c}0.142 \\
48\end{array}$ & $\begin{array}{c}2.2869 \\
7 *\end{array}$ & $\begin{array}{c}4.8211 \\
9\end{array}$ & $\begin{array}{c}10.6300 \\
7 *\end{array}$ & 0.03784 & 0.55209 & $\begin{array}{c}1.7873 \\
3\end{array}$ & $\begin{array}{c}3.58181 \\
*\end{array}$ & $\begin{array}{c}0.1973 \\
7\end{array}$ & $\begin{array}{c}3.13899 \\
*\end{array}$ & $\begin{array}{c}7.5414 \\
2\end{array}$ & $\begin{array}{c}16.4750 \\
8^{*}\end{array}$ \\
\hline 23 & $\begin{array}{c}0.125 \\
05\end{array}$ & $\begin{array}{c}2.5874 \\
1 *\end{array}$ & $\begin{array}{c}4.9462 \\
4\end{array}$ & $\begin{array}{c}13.9269 \\
1 *\end{array}$ & 0.00811 & $\left|\begin{array}{c}- \\
0.14817\end{array}\right|$ & $\begin{array}{c}1.7792 \\
3\end{array}$ & $\begin{array}{c}4.42628 \\
*\end{array}$ & $\begin{array}{c}0.1790 \\
9\end{array}$ & $\begin{array}{c}3.63030 \\
*\end{array}$ & $\begin{array}{c}7.7205 \\
1\end{array}$ & $\begin{array}{c}21.2968 \\
1^{*}\end{array}$ \\
\hline 24 & $\begin{array}{c}0.138 \\
20\end{array}$ & $\begin{array}{c}2.7444 \\
1 *\end{array}$ & $\begin{array}{c}5.0844 \\
5\end{array}$ & $\begin{array}{c}13.6140 \\
8^{*}\end{array}$ & 0.07998 & 1.48316 & $\begin{array}{c}1.8592 \\
1\end{array}$ & $\begin{array}{c}4.64884 \\
*\end{array}$ & $\begin{array}{c}0.1912 \\
2\end{array}$ & $\begin{array}{c}3.55034 \\
*\end{array}$ & $\begin{array}{c}7.9117 \\
3\end{array}$ & $\begin{array}{c}19.8072 \\
5^{*}\end{array}$ \\
\hline
\end{tabular}




\begin{tabular}{|c|c|c|c|c|c|c|c|c|c|c|c|c|}
\hline 25 & $\begin{array}{c}0.195 \\
28\end{array}$ & $\begin{array}{c}2.7985 \\
3^{*}\end{array}$ & $\begin{array}{c}5.2797 \\
3\end{array}$ & $\begin{array}{c}10.1106 \\
5^{*}\end{array}$ & 0.13695 & 1.82718 & $\begin{array}{c}1.9961 \\
6\end{array}$ & $\begin{array}{c}3.55895 \\
*\end{array}$ & $\begin{array}{c}0.2451 \\
7\end{array}$ & $\begin{array}{c}3.45987 \\
*\end{array}$ & $\begin{array}{c}8.1569 \\
0\end{array}$ & $\begin{array}{c}15.3823 \\
2^{*}\end{array}$ \\
\hline 26 & $\begin{array}{c}0.020 \\
75\end{array}$ & $\begin{array}{c}0.3541 \\
0\end{array}$ & $\begin{array}{c}5.3004 \\
8\end{array}$ & $\begin{array}{c}11.9805 \\
5^{*}\end{array}$ & 0.03229 & 0.54292 & $\begin{array}{c}2.0284 \\
5\end{array}$ & $\begin{array}{c}4.51728 \\
*\end{array}$ & $\begin{array}{c}0.0722 \\
6\end{array}$ & 1.19352 & $\begin{array}{c}8.2291 \\
6\end{array}$ & $\begin{array}{c}18.0041 \\
8^{*}\end{array}$ \\
\hline 27 & $\begin{array}{c}- \\
0.015 \\
70\end{array}$ & $\begin{array}{c}- \\
0.3218 \\
4\end{array}$ & $\begin{array}{c}5.2847 \\
8\end{array}$ & $\begin{array}{c}14.2204 \\
6^{*}\end{array}$ & 0.00532 & 0.10453 & $\begin{array}{c}2.0337 \\
7\end{array}$ & $\begin{array}{c}5.24491 \\
*\end{array}$ & $\begin{array}{c}0.0380 \\
3\end{array}$ & 0.76067 & $\begin{array}{c}8.2671 \\
9\end{array}$ & $\begin{array}{c}21.7134 \\
2^{*}\end{array}$ \\
\hline 28 & $\begin{array}{c}0.032 \\
06\end{array}$ & $\begin{array}{c}0.6015 \\
4\end{array}$ & $\begin{array}{c}5.2527 \\
1\end{array}$ & $\begin{array}{c}12.8291 \\
2^{*}\end{array}$ & -0.01578 & $\begin{array}{c}- \\
0.28175\end{array}$ & $\begin{array}{c}2.0180 \\
0\end{array}$ & $\begin{array}{c}4.69154 \\
*\end{array}$ & $\begin{array}{c}0.0232 \\
3\end{array}$ & 0.42195 & $\begin{array}{c}8.2904 \\
2\end{array}$ & $\begin{array}{c}19.6032 \\
6^{*}\end{array}$ \\
\hline 29 & $\begin{array}{c}0.170 \\
32\end{array}$ & $\begin{array}{c}3.0834 \\
6^{*}\end{array}$ & $\begin{array}{c}5.4230 \\
3\end{array}$ & $\begin{array}{c}12.6748 \\
9 *\end{array}$ & 0.10115 & 1.71521 & $\begin{array}{c}2.1191 \\
4\end{array}$ & $\begin{array}{c}4.63927 \\
*\end{array}$ & $\begin{array}{c}0.2236 \\
0\end{array}$ & $\begin{array}{c}3.93251 \\
*\end{array}$ & $\begin{array}{c}8.5140 \\
2\end{array}$ & $\begin{array}{c}19.3314 \\
4 *\end{array}$ \\
\hline 30 & $\begin{array}{c}0.016 \\
79\end{array}$ & $\begin{array}{c}0.3363 \\
9\end{array}$ & $\begin{array}{c}5.4398 \\
2\end{array}$ & $\begin{array}{c}13.9524 \\
6^{*}\end{array}$ & 0.04568 & 0.84802 & $\begin{array}{c}2.1648 \\
2\end{array}$ & $\begin{array}{c}5.14547 \\
*\end{array}$ & $\begin{array}{c}0.0704 \\
9\end{array}$ & 1.37442 & $\begin{array}{c}8.5845 \\
1\end{array}$ & $\begin{array}{c}21.4312 \\
1 *\end{array}$ \\
\hline
\end{tabular}

Note: * Indicates Statistically Significant at 5\% Level of Significance

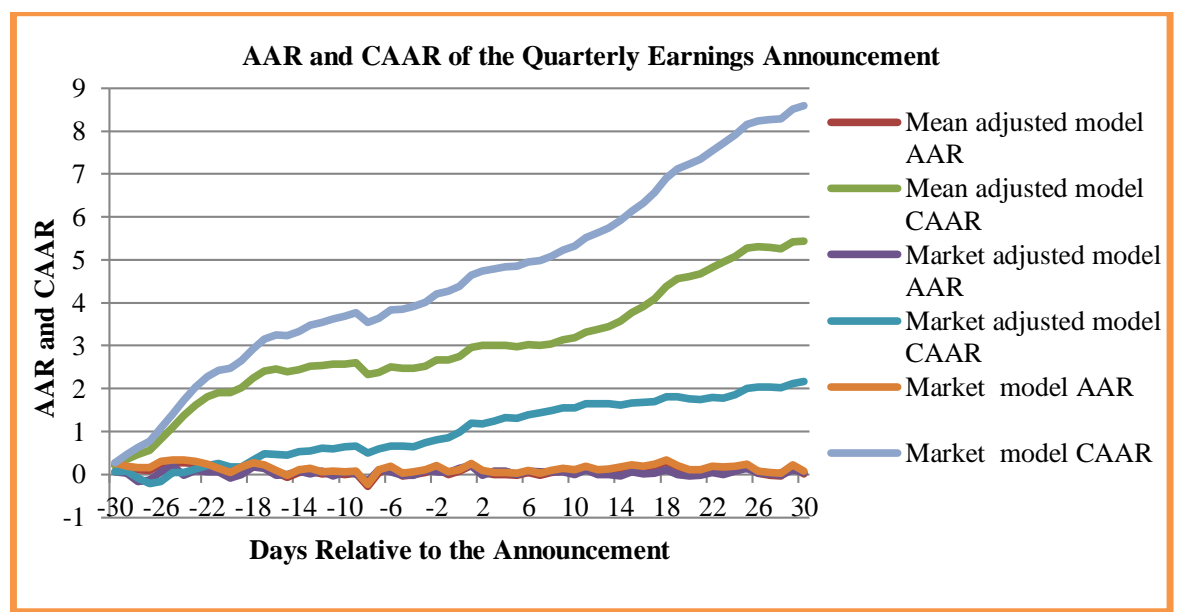

Figure 2 AARs and CAARs Trends of Three Models over the 61-Day Event Window of Good News Earnings Announcement of September 2012 Quarter

Table 3 and Figure 3 present the AAR and CAAR values of bad sample earnings announcement of mean adjusted model, market adjusted model and market model of September -2012 quarter. In the case of mean adjusted model and market model, the AAR are positive and significant for majority of the days in the event window of 61 days. The AARs of market adjusted model are positive and insignificant. This result indicates that earnings announcement contains information which help the traders to gain abnormal returns. In contrast, for day 0 , AAR is negative and significant and market responded negatively on the day of announcement. The CAAR values of mean adjusted model and market model are positive and significant throughout the event window. This shows that the market participant can act on quarterly earnings information to earn an average abnormal return during the event window. Hence we reject the null hypothesis that CAAR values are close to zero. In the case of market adjusted model, the CAARs are positive for 61 days and insignificant for 36 days. Therefore, we accept the null hypothesis that CAARs are close to zero. 
Table $3 A A R$ and CAAR Values of Bad News Earnings Announcements

\begin{tabular}{|c|c|c|c|c|c|c|c|c|c|c|c|c|}
\hline \multirow[t]{2}{*}{ Days } & \multicolumn{4}{|c|}{ Mean adjusted model } & \multicolumn{4}{|c|}{ Market adjusted model } & \multicolumn{4}{|c|}{ Market model } \\
\hline & AAR & & AAR & le & $\mathbf{A R}$ & & AAR & e & $\mathbf{R}$ & t value & CAAR & \\
\hline-30 & 0.30408 & & 408 & & 73 & & & & & & & \\
\hline-29 & 0.17704 & & & & & & & & & & & \\
\hline-28 & 0.41226 & 6.21 & 9338 & $*$ & 17248 & $6 *$ & 2859 & $92 *$ & 85 & $62 *$ & 019 & 8. \\
\hline-27 & 0.2 & & 100 & & $\begin{array}{c}- \\
0.00131 \\
\end{array}$ & & & & & & 97 & \\
\hline-26 & 0.1 & 2.76 & 1.27344 & & $\begin{array}{c}- \\
0.01898 \\
\end{array}$ & 43 & 830 & $42 *$ & 097 & $87 *$ & 994 & 9.7 \\
\hline-25 & 0.05401 & 0.90500 & 1.32745 & $4 *$ & $\begin{array}{c}- \\
0.07129 \\
\end{array}$ & -1.06331 & 3701 & 1.44320 & 0.08027 & 1.30726 & 1.48021 & $97 *$ \\
\hline-24 & 313 & & 9058 & & 0.08400 & 59 & 100 & 386 & & $32 *$ & 1.86507 & 9 \\
\hline-23 & 0.28956 & & & & 773 & & & & & $18 *$ & & \\
\hline-22 & 0.15577 & 2.3 & 92 & & 23 & & & & & $19 *$ & & 1 \\
\hline-21 & 0.14552 & & 2.28144 & & 0.08301 & & & & & $36 *$ & & 12 \\
\hline-20 & 0.18272 & 2.87 & 2.46416 & & 2044 & 1.76777 & 40 & $98 *$ & 049 & $31 *$ & & 345 \\
\hline 19 & 53 & & & & $\begin{array}{c}- \\
0.05899\end{array}$ & & & & 906 & 67 & 968 & $29 \%$ \\
\hline-18 & 992 & 19 & 2.6 & & $\mid \begin{array}{c}- \\
0.03252\end{array}$ & &  & $19 *$ & 202 & 860 & 70 & 12 \\
\hline-17 & 381 & & 442 & & 0.07338 & & & & & $00 *$ & & \\
\hline-16 & 883 & & & & $\begin{array}{c}- \\
0.04265 \\
\end{array}$ & & & $75 *$ & 467 & 303 & 10 & 13 \\
\hline-15 & 975 & & 500 & & 3471 & & & & 806 & 55 & & \\
\hline-14 & 0.03211 & & 288 & & 0.03839 & & & $76 *$ & $\begin{array}{c}- \\
0.01103\end{array}$ & 686 & & \\
\hline-13 & 0.14430 & & 19 & 10.7 & 434 & & & & 41 & $31 *$ & 54 & \\
\hline-12 & \begin{tabular}{|c|}
- \\
0.04143 \\
\end{tabular} & & 576 & & 0.01389 & & & $4 *$ & 0.00263 & 81 & 692 & \\
\hline-11 & 0.14271 & & 46 & & 587 & & & & 86 & $5 \%$ & & \\
\hline-10 & 0.00221 & 03842 & 5067 & 11.59 & 0.02692 & 0.46620 & 0.85520 & $68 *$ & 0.02107 & & & \\
\hline-9 & $\begin{array}{c}- \\
0.06032 \\
\end{array}$ & & 035 & 11.4 & $\begin{array}{c}- \\
0.06360\end{array}$ & & 60 & $97 *$ & $\begin{array}{c}- \\
0.04731 \\
\end{array}$ & 114 & 154 & \\
\hline-8 & \begin{tabular}{|c|}
- \\
0.05588 \\
\end{tabular} & 065 & 3447 & 11 & 0.03194 & 54 & 54 & $87 *$ & $\begin{array}{c}- \\
0.04296 \\
\end{array}$ & 75 & 858 & $81 *$ \\
\hline-7 & $\begin{array}{c}- \\
0.05051\end{array}$ & -0 & 8396 & $6^{*}$ & 0.03569 & 18 & 85 & $32 *$ & $\begin{array}{c}- \\
0.03152 \\
\end{array}$ & 135 & 3.39706 & $46^{*}$ \\
\hline-6 & 0.02306 & 0.40593 & 2.90702 & $23319^{*}$ & $\begin{array}{c}- \\
0.05304\end{array}$ & -0.93698 & 481 & $2.59623 *$ & 0.05900 & 0.99051 & 3.45606 & $388 *$ \\
\hline-5 & \begin{tabular}{|c|}
- \\
0.12296 \\
\end{tabular} & $\begin{array}{c}- \\
2.52853 * \\
\end{array}$ & 2.78406 & $11.22792 *$ & $\begin{array}{c}- \\
0.13972 \\
\end{array}$ & $\begin{array}{c}- \\
2.71598 * \\
\end{array}$ & & $62 *$ & $\begin{array}{c}- \\
0.09167 \\
\end{array}$ & -1.65772 & 3.36439 & \\
\hline-4 & \begin{tabular}{|c|}
- \\
0.02782 \\
\end{tabular} & -0.49929 & 2.75624 & $9.51915^{*}$ & $\begin{array}{c}- \\
0.05352 \\
\end{array}$ & -0.97270 & 157 & 1.89415 & $\begin{array}{c}- \\
0.00962 \\
\end{array}$ & -0.16533 & 3.35477 & 11. \\
\hline-3 & 0.05453 & 1.07526 & 2.81077 & $10.47391 *$ & 0.05100 & 0.98251 & 0.59257 & $2.15737 *$ & 0.06266 & 1.13382 & 3.41744 & \\
\hline-2 & 0.14821 & $2.80890 *$ & 398 & $10.41381 *$ & 088 & 1.34911 & 0.66 & $89 * 0$ & 0.16401 & $2.94920 *$ & & $11.95884 *$ \\
\hline 1 & 0.08422 & 1.46908 & 3.04320 & $9.69115^{*}$ & 0.10951 & 1.89463 & 0.77296 & $2.44153 *$ & 0.11636 & 1.96835 & 3.69781 & $11.42035^{*}$ \\
\hline
\end{tabular}




\begin{tabular}{|c|c|c|c|c|c|c|c|c|c|c|c|c|}
\hline 0 & $\begin{array}{c}- \\
0.32540 \\
\end{array}$ & $\begin{array}{c}- \\
3.45171 *\end{array}$ & 2.71780 & $5.17781 *$ & $\begin{array}{c}- \\
0.28616 \\
\end{array}$ & $2.91031 *$ & 0.48680 & 0.88920 & \begin{tabular}{|c|}
- \\
0.32257 \\
\end{tabular} & 3. & 3.37524 & $6.22384 *$ \\
\hline 1 & $\begin{array}{c}- \\
0.09118 \\
\end{array}$ & -1.08833 & 2.62662 & $5.54231 *$ & $\begin{array}{c}- \\
0.09897 \\
\end{array}$ & -1.08574 & 0.38783 & 0.75210 & $\begin{array}{c}- \\
0.07679 \\
\end{array}$ & -0.87947 & 3.29845 & $6.67784 *$ \\
\hline 2 & 0.02178 & 0.43967 & 2.64840 & $9.30754 *$ & $\begin{array}{c}- \\
0.03225\end{array}$ & -0.60716 & 0.35558 & 1.16542 & 0.03453 & 0.64157 & 3.33298 & $10.77867 *$ \\
\hline 3 & $\begin{array}{c}- \\
0.05332\end{array}$ & -0.98543 & 2.59507 & 8.2 & $\begin{array}{c}- \\
0.00396\end{array}$ & -0.06946 & 162 & 43 & $0 . \overline{-}$ & 351 & 396 & \\
\hline 4 & $\begin{array}{c}- \\
0.08533 \\
\end{array}$ & -1.57302 & 2.50974 & $7.82046^{*}$ & $\begin{array}{c}- \\
0.03152 \\
\end{array}$ & -0.54715 & 0.32010 & 0.93909 & $\begin{array}{c}- \\
0.07292 \\
\end{array}$ & -1.26230 & 105 & 9.4 \\
\hline 5 & 0.00256 & 0.04853 & 2.51230 & $7.94385 *$ & 0.02121 & 0.39884 & 0.34131 & 1.06949 & 0.02766 & 0.47811 & 3.24871 & 9.35783* \\
\hline 6 & $\begin{array}{c}- \\
0.07978\end{array}$ & -1.61836 & 2.43252 & $8.11195 *$ & $\begin{array}{c}- \\
0.05440\end{array}$ & -1.09632 & 0.28691 & 0.95061 & $\begin{array}{c}- \\
0.06049\end{array}$ & -1.12854 & 3.18822 & 9.77 \\
\hline 7 & \begin{tabular}{|c|}
- \\
0.13960 \\
\end{tabular} & $\begin{array}{c}- \\
2.80565^{*}\end{array}$ & 2.29292 & $7.47541 *$ & $\begin{array}{c}- \\
0.12449 \\
\end{array}$ & \begin{tabular}{c|}
- \\
$2.44647 *$ \\
\end{tabular} & 0.16242 & 0.51777 & \begin{tabular}{|c|}
- \\
0.10417 \\
\end{tabular} & -1.93802 & 3.08404 & $9.30766^{*}$ \\
\hline 8 & $\begin{array}{c}- \\
0.09549 \\
\end{array}$ & -1.64872 & 2.19743 & $6.07566^{*}$ & $\begin{array}{c}- \\
0.12536 \\
\end{array}$ & $\begin{array}{c}- \\
2.04716 * \\
\end{array}$ & 0.03705 & 0.09689 & \begin{tabular}{|c|}
- \\
0.07446 \\
\end{tabular} & -1.21007 & 3.00958 & $7.83154 *$ \\
\hline 9 & 0.09292 & 1.77076 & 2.29035 & $6.90127 *$ & 0.04484 & 0.84047 & 0.08190 & 0.24270 & 0.10408 & 1.87787 & 3.11366 & $8.88288^{*}$ \\
\hline 10 & 0.13571 & 1.63078 & 2.42605 & $4.55309 *$ & 0.05116 & 0.63666 & 0.13306 & 0.25860 & 0.14803 & 1.87857 & 3.26169 & $6.46449 *$ \\
\hline 11 & 0.07764 & 1.40625 & 2.50369 & 6.99 & 0.00641 & 0.11407 & 9947 & 0.38292 & 0.10307 & 1.72468 & 3.36476 & $77^{*}$ \\
\hline 12 & 0.03314 & 0.56205 & 2.53684 & 6.5 & $\begin{array}{c}- \\
0.07411 \\
\end{array}$ & -1.21370 & 536 & 323 & 0.03105 & 0.49452 & 580 & $29 *$ \\
\hline 13 & 0.06425 & 0.9 & 2.60109 & $8 *$ & $\begin{array}{c}- \\
0.04140 \\
\end{array}$ & 38 & 96 & 52 & 0.08078 & 923 & 658 & $3 *$ \\
\hline 14 & 0.21829 & $3.07938 *$ & 2.8 & 5.92 & 0.05962 & 0.74460 & 0.08358 & 0.15561 & 0.22745 & $77 *$ & 3.70403 & $24 *$ \\
\hline 15 & 0.19223 & $3.00589 *$ & 3.01161 & $6.94328 *$ & 0.06262 & 0.92936 & 4620 & 0.31992 & 0.18919 & 2.77 & 3.89322 & $778^{*}$ \\
\hline 16 & 0.09695 & 1.50885 & 3.10856 & $7.05676^{*}$ & $\begin{array}{c}- \\
0.02996 \\
\end{array}$ & -0.42721 & 0.11623 & 0.24174 & 0.11710 & 1.73125 & 4.01031 & $8.64842 *$ \\
\hline 17 & 0.13938 & $2.53135^{*}$ & $=3.24795$ & $91 *$ & $\begin{array}{c}- \\
0.03289 \\
\end{array}$ & -0.55941 & 0.08335 & 0.20465 & 0.16499 & $2.83185^{*}$ & 531 & $57 *$ \\
\hline 18 & $0.18482 \mid$ & $3.06458 *$ & 3.43277 & $8.13142 *$ & $\begin{array}{c}- \\
0.02144\end{array}$ & -0.3 & 0.06191 & 0.13612 & 0.21026 & $3.44919 *$ & 557 & $759 *$ \\
\hline 19 & 0.29660 & 4.47 & 3.72936 & 7.96 & 0.14484 & $2.10460 *$ & 676 & 486 & 0.30913 & 4. & 470 & 10. \\
\hline 20 & 0.17431 & $3.09407 *$ & 3.90367 & $9.70284 *$ & 0.07909 & 1.38513 & 585 & 097 & 0.18509 & $3.12864 *$ & 4.87979 & $11.54996^{*}$ \\
\hline 21 & 0.11140 & 1.78889 & 4.01507 & $8.94139 *$ & 0.02270 & 0.36148 & 0.30855 & 0.68139 & 0.13895 & $2.19040 *$ & 5.01875 & $10.97093 *$ \\
\hline 22 & 0.16099 & $2.92148 *$ & 4.17606 & $10.40929 *$ & 0.04242 & 0.75604 & 5097 & 0.85920 & 0.18923 & $3.25774 *$ & 5.20798 & $564 *$ \\
\hline 23 & 0.13420 & $2.20351 *$ & 4.31026 & $9.63082 *$ & 0.00651 & -0.10286 & 0.34445 & 0.74008 & 0.15625 & $2.51891 *$ & 5.36423 & $11.76782 *$ \\
\hline 24 & 0.07147 & 1.05918 & 4.38173 & $8.75602 *$ & $\begin{array}{c}- \\
0.00482 \\
\end{array}$ & -0.06731 & 0.33963 & 0.63924 & 0.10196 & 1.48988 & 5.46619 & $10.77014 *$ \\
\hline 25 & 0.05372 & 0.87210 & 4.43546 & $9.62159 *$ & $\begin{array}{c}- \\
0.03864 \\
\end{array}$ & -0.60308 & 0.30099 & 0.62777 & 0.07995 & 1.28897 & 5.54614 & $11.94833^{*}$ \\
\hline 26 & 0.06336 & 1.17317 & 4.49881 & $11.03378 *$ & 0.04336 & 0.75745 & 0.34435 & 0.79673 & 0.09600 & 1.70145 & 5.64215 & $13.24446^{*}$ \\
\hline 27 & 0.00415 & 0.06715 & 4.50297 & $9.56004 *$ & 0.00938 & 0.15220 & 0.35373 & 0.75384 & 0.02467 & 0.39921 & 5.66682 & $12.04036^{*}$ \\
\hline 28 & \begin{tabular}{|c|}
- \\
0.02068 \\
\end{tabular} & -0.32022 & 4.48229 & $9.03504 *$ & $\begin{array}{c}- \\
0.01583 \\
\end{array}$ & 923 & 0.33790 & 0.66483 & 0.01297 & -0.19912 & 5.65384 & $11.29692 *$ \\
\hline 29 & 0.03980 & 0.81083 & 4.52209 & $11.89332 *$ & $\mid \begin{array}{c}- \\
0.02771\end{array}$ & -0.55004 & 0.31020 & 0.79500 & 0.05024 & 0.93690 & 5.70409 & $13.73140 *$ \\
\hline 30 & $\begin{array}{c}- \\
0.01831 \\
\end{array}$ & -0.36964 & 4.50378 & $11.64267 *$ & $\begin{array}{c}- \\
0.03009 \\
\end{array}$ & -0.58771 & 0.28010 & 0.70048 & 0.01750 & 0.32781 & 5.72159 & $13.72072 *$ \\
\hline
\end{tabular}

Note: * Indicates Statistically Significant at 5\% Level Of Significance. 


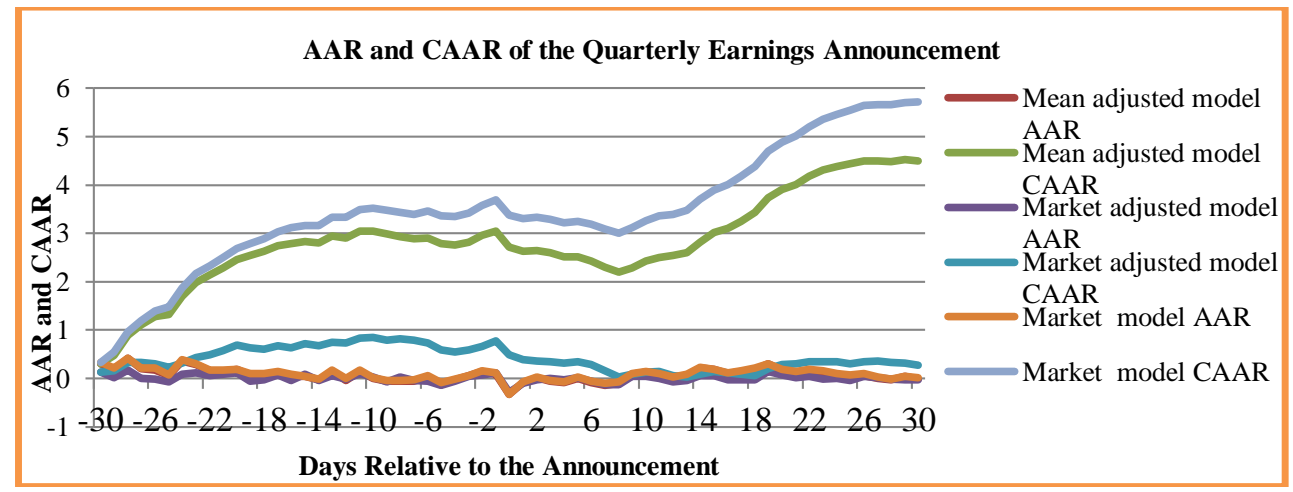

Figure 3 AARs and CAARs Trends of Three Models over the 61-Day Event Window of Bad News Earnings Announcement of September 2012 Quarter

Table 4 Runs and Sign Test Statistics of September 2012 Quarter

\begin{tabular}{|c|c|c|c|c|c|c|}
\hline & Mean adjusted model & Market adjusted model & \multicolumn{2}{c|}{ Market model } \\
\hline & $\begin{array}{c}\text { Runs } \\
\text { Statistics }\end{array}$ & $\begin{array}{c}\text { Sign } \\
\text { Statistics }\end{array}$ & $\begin{array}{c}\text { Runs } \\
\text { Statistics }\end{array}$ & $\begin{array}{c}\text { Sign } \\
\text { Statistics }\end{array}$ & $\begin{array}{c}\text { Runs } \\
\text { Statistics }\end{array}$ & $\begin{array}{c}\text { Sign } \\
\text { Statistics }\end{array}$ \\
\hline \multicolumn{7}{|c|}{ Good News Earnings Announcement } \\
\hline Before & -2.9729 & 4.9934 & 0.4795 & 2.4327 & -2.9729 & 7.2981 \\
\hline After & -1.9136 & 3.6515 & -0.5427 & 1.4606 & -1.9136 & 4.7469 \\
\hline Overall & -3.4569 & 3.4125 & 0.4074 & 1.9757 & -3.4569 & 5.5678 \\
\hline \multicolumn{7}{|c|}{ Bad News Earnings Announcement } \\
\hline Before & -1.4684 & 3.7131 & 0.3982 & -0.3841 & -1.4684 & 3.9691 \\
\hline After & -4.1891 & 2.9212 & -1.2742 & 0.7303 & -4.1891 & 2.9212 \\
\hline Overall & -4.5182 & 2.3349 & -1.4047 & -1.2572 & -3.9934 & 2.6941 \\
\hline \multicolumn{7}{|c|}{ Full Sample Earnings Announcement } \\
\hline Before & -2.9729 & 3.7131 & -1.0348 & 3.2009 & -2.9729 & 6.5299 \\
\hline After & -3.4512 & 3.2863 & 0.1888 & 2.5560 & -3.4512 & 4.7469 \\
\hline Overall & -4.4969 & 1.9757 & -0.3692 & 1.9757 & -3.9518 & 4.4901 \\
\hline
\end{tabular}

\section{Notes:}

1. Before: Number of Runs, Run Statistics, and Sign Statistics before the event day.

2. After: Number of Runs, Run Statistics, and Sign Statistics after the event day.

3. Overall: Number of Runs, Run Statistics, and Sign Statistics for the event window (-30 through 30 days.)

4. If the Run and Sign test statistics is greater than the critical value of \pm 1.96 , the relevant AAR is statistically significant at 5\% level of Significance.

It is observed that the AARs of mean adjusted model and market model of all the portfolios are significant for overall period and therefore, we reject the null hypothesis that AARs occur randomly at $5 \%$ level of significance for the entire event window. Whereas, the result of market adjusted model shows that AARs are insignificant for all the portfolios in the event window of 61 days. Therefore, we accept that AARs are random under this model. The sign test on AAR is presented in Table 4 for all the three portfolios. The values of sign statistics of mean adjusted model, market adjusted model and market model are greater than the critical value of 
\pm 1.96 for overall sample during the window period of 61 days. Therefore, we conclude that there is a significant difference between the number of positive and negative AARs.

The estimated beta and $\mathrm{R}^{2}$ from market model are presented in Table 5 . The average betas are negatively changed during pre and post event period for all the length intervals except first two days. The first pass beta ranges from 0.8277 to 1.2587 during the pre-event and from 0.8956 to 0.9997 for the post-event period. Using one day return interval betas rise to $8.46 \%$. With two days interval, betas rise $4.44 \%, 5^{\text {th }}$ day interval fall to $-15.43 \%, 10^{\text {th }}$ day interval fall to $-14.31 \%, 15^{\text {th }}$ day interval fall to $-19.80 \%$, and on $20^{\text {th }}$ day interval fall to $-20.58 \%$. The proportionate decrease in beta shows poor price adjustment on the quarterly earnings announcement and indicates poor market quality. The price efficiency is observed by $\mathrm{R}^{2}$ in the market model regression. In the case of $\mathrm{R}^{2}$, positive change is observed for $2^{\text {nd }}$ to $8^{\text {th }}$ and $19^{\text {th }}$ day. The remaining days, $R^{2}$ are negatively changed The $R^{2}$ values ranges from 0.1183 to 0.4000 for the pre event period and from 0.1054 to 0.3805 for the post event period. The highest positive change of $36.71 \%$ is observed on the $3^{\text {rd }}$ day interval period. The $\mathrm{R}^{2}$ values are decreased proportionately during the post event period which is not expected in the study. This shows price inefficiency. The Table 6 shows the result of second pass beta. The average BETA2 parameter should be less negative when market frictions are less. So, we expect a positive change in BETA2 during the earnings announcement. The BETA2 are positively changed for all intervals. The BETA2 are negatively signed in the post event period and this shows less market frictions in the market.

Table 5 The Results of First Pass Beta and R Square Coefficients

\begin{tabular}{|c|c|c|c|c|c|c|c|c|c|c|c|c|}
\hline \multirow{3}{*}{$\begin{array}{c}\text { Leng } \\
\text { th } \\
\text { Inter } \\
\text { vals }\end{array}$} & \multicolumn{6}{|c|}{ Beta } & \multicolumn{6}{|c|}{ R Square } \\
\hline & \multicolumn{2}{|c|}{ Pre-Event } & \multicolumn{2}{|c|}{ Post-Event } & \multirow[b]{2}{*}{$\begin{array}{l}\text { Chan } \\
\text { ge in } \\
\text { Beta }\end{array}$} & \multirow{2}{*}{$\begin{array}{c}\% \\
\text { Cha } \\
\text { nge } \\
\text { in } \\
\text { Beta }\end{array}$} & \multicolumn{2}{|c|}{ Pre-Event } & \multicolumn{2}{|c|}{ Post-Event } & \multirow{2}{*}{$\begin{array}{c}\text { Chan } \\
\text { ge in } \\
\mathbf{R} \\
\text { Squa } \\
\text { re }\end{array}$} & \multirow{2}{*}{$\begin{array}{c}\% \\
\text { Cha } \\
\text { nge } \\
\text { in R } \\
\text { Squ } \\
\text { are }\end{array}$} \\
\hline & $\begin{array}{c}\text { Aver } \\
\text { age }\end{array}$ & $\begin{array}{c}\text { STD } \\
\text { EV }\end{array}$ & $\begin{array}{l}\text { Aver } \\
\text { age }\end{array}$ & $\begin{array}{c}\text { STD } \\
\text { EV }\end{array}$ & & & $\begin{array}{c}\text { Aver } \\
\text { age }\end{array}$ & $\begin{array}{c}\text { STD } \\
\text { EV }\end{array}$ & $\begin{array}{l}\text { Aver } \\
\text { age }\end{array}$ & $\begin{array}{c}\text { STD } \\
\text { EV }\end{array}$ & & \\
\hline 1 & $\begin{array}{c}0.827 \\
682\end{array}$ & $\begin{array}{c}0.838 \\
831\end{array}$ & $\begin{array}{c}0.897 \\
727\end{array}$ & $\begin{array}{c}0.793 \\
018\end{array}$ & $\begin{array}{c}0.070 \\
045\end{array}$ & $\begin{array}{c}8.46 \\
\%\end{array}$ & $\begin{array}{c}0.118 \\
338\end{array}$ & $\begin{array}{c}0.163 \\
815\end{array}$ & $\begin{array}{c}0.105 \\
425\end{array}$ & $\begin{array}{c}0.139 \\
608\end{array}$ & $\begin{array}{c}0.012 \\
91\end{array}$ & $\begin{array}{c}10.9 \\
1 \%\end{array}$ \\
\hline 2 & $\begin{array}{c}0.870 \\
697 \\
\end{array}$ & \begin{tabular}{|c|}
1.045 \\
266 \\
\end{tabular} & $\begin{array}{c}0.909 \\
365 \\
\end{array}$ & $\begin{array}{r}81 \\
\end{array}$ & $\begin{array}{c}0.038 \\
669 \\
\end{array}$ & $\begin{array}{c}4.44 \\
\%\end{array}$ & $\begin{array}{c}0.128 \\
418 \\
\end{array}$ & $\begin{array}{c}0.170 \\
482 \\
\end{array}$ & $\begin{array}{c}0.163 \\
685 \\
\end{array}$ & $\begin{array}{c}0.178 \\
369 \\
\end{array}$ & $\begin{array}{c}0.035 \\
268 \\
\end{array}$ & $\begin{array}{c}27.4 \\
6 \% \\
\end{array}$ \\
\hline 3 & $\begin{array}{c}0.943 \\
399\end{array}$ & $\begin{array}{c}1.100 \\
731\end{array}$ & $\begin{array}{c}0.920 \\
8\end{array}$ & $\begin{array}{c}0.866 \\
205\end{array}$ & $\begin{array}{c}0.022 \\
6\end{array}$ & $\begin{array}{c}2.40 \\
\%\end{array}$ & $\begin{array}{c}0.151 \\
18\end{array}$ & $\begin{array}{c}0.183 \\
446\end{array}$ & $\begin{array}{c}0.206 \\
681\end{array}$ & $\begin{array}{c}0.204 \\
398\end{array}$ & $\begin{array}{c}0.055 \\
501\end{array}$ & $\begin{array}{c}36.7 \\
1 \%\end{array}$ \\
\hline 4 & $\begin{array}{c}1.021 \\
489\end{array}$ & $\begin{array}{c}1.200 \\
426\end{array}$ & $\begin{array}{c}0.913 \\
97\end{array}$ & $\begin{array}{c}0.906 \\
984\end{array}$ & $\begin{array}{c}- \\
0.107 \\
52 \\
\end{array}$ & $\begin{array}{c}- \\
10.5 \\
3 \%\end{array}$ & $\begin{array}{c}0.184 \\
057\end{array}$ & $\begin{array}{c}0.201 \\
067\end{array}$ & $\begin{array}{c}0.241 \\
72\end{array}$ & $\begin{array}{c}0.225 \\
029\end{array}$ & $\begin{array}{c}0.057 \\
663\end{array}$ & $\begin{array}{c}31.3 \\
3 \%\end{array}$ \\
\hline 5 & $\begin{array}{c}1.076 \\
822\end{array}$ & $\begin{array}{c}1.350 \\
808\end{array}$ & $\begin{array}{c}0.910 \\
645\end{array}$ & $\begin{array}{c}0.917 \\
793\end{array}$ & $\begin{array}{c}0.166 \\
18 \\
\end{array}$ & $\begin{array}{l}15.4 \\
3 \%\end{array}$ & $\begin{array}{c}0.209 \\
599\end{array}$ & $\begin{array}{c}0.220 \\
005\end{array}$ & $\begin{array}{c}0.268 \\
652\end{array}$ & $\begin{array}{c}0.239 \\
493\end{array}$ & $\begin{array}{c}0.059 \\
054\end{array}$ & $\begin{array}{l}28.1 \\
7 \%\end{array}$ \\
\hline 6 & $\begin{array}{c}1.105 \\
882\end{array}$ & $\begin{array}{c}1.464 \\
644\end{array}$ & $\begin{array}{c}0.902 \\
75\end{array}$ & $\begin{array}{c}0.940 \\
817\end{array}$ & $\begin{array}{c}- \\
0.203 \\
13\end{array}$ & $\begin{array}{c}18.3 \\
7 \%\end{array}$ & $\begin{array}{c}0.237 \\
606\end{array}$ & $\begin{array}{c}0.237 \\
043\end{array}$ & $\begin{array}{c}0.284 \\
261\end{array}$ & $\begin{array}{c}0.251 \\
785\end{array}$ & $\begin{array}{c}0.046 \\
655\end{array}$ & $\begin{array}{c}19.6 \\
4 \%\end{array}$ \\
\hline
\end{tabular}




\begin{tabular}{|c|c|c|c|c|c|c|c|c|c|c|c|c|}
\hline 7 & $\begin{array}{c}1.127 \\
962\end{array}$ & $\begin{array}{c}1.608 \\
41\end{array}$ & $\begin{array}{c}0.895 \\
567\end{array}$ & $\begin{array}{c}0.969 \\
874\end{array}$ & $\begin{array}{c}0.232 \\
4\end{array}$ & $\begin{array}{c}20.6 \\
0 \%\end{array}$ & $\begin{array}{c}0.260 \\
086\end{array}$ & $\begin{array}{c}0.255 \\
901\end{array}$ & $\begin{array}{c}0.291 \\
978\end{array}$ & $\begin{array}{c}0.261 \\
065\end{array}$ & $\begin{array}{c}0.031 \\
892\end{array}$ & $\begin{array}{c}12.2 \\
6 \%\end{array}$ \\
\hline 8 & $\begin{array}{c}1.139 \\
45\end{array}$ & $\begin{array}{c}1.658 \\
023\end{array}$ & $\begin{array}{c}0.901 \\
409\end{array}$ & $\begin{array}{c}1.003 \\
581\end{array}$ & $\begin{array}{c}0.238 \\
04\end{array}$ & $\begin{array}{c}20.8 \\
9 \%\end{array}$ & $\begin{array}{c}0.286 \\
468\end{array}$ & $\begin{array}{c}0.267 \\
79\end{array}$ & $\begin{array}{c}0.300 \\
205\end{array}$ & $\begin{array}{c}0.266 \\
662\end{array}$ & $\begin{array}{c}0.013 \\
737\end{array}$ & $\begin{array}{c}4.80 \\
\%\end{array}$ \\
\hline 9 & $\begin{array}{c}1.129 \\
095\end{array}$ & $\begin{array}{c}1.796 \\
999\end{array}$ & $\begin{array}{c}0.917 \\
677\end{array}$ & $\begin{array}{c}1.051 \\
684\end{array}$ & $\begin{array}{c}- \\
0.211 \\
42\end{array}$ & $\begin{array}{c}- \\
18.7 \\
2 \%\end{array}$ & $\begin{array}{c}0.308 \\
904\end{array}$ & $\begin{array}{c}0.279 \\
581\end{array}$ & $\begin{array}{c}0.308 \\
076\end{array}$ & $\begin{array}{c}0.271 \\
919\end{array}$ & $\begin{array}{c}- \\
0.000 \\
83\end{array}$ & $\begin{array}{c}- \\
0.27 \\
\%\end{array}$ \\
\hline 10 & $\begin{array}{c}1.075 \\
441\end{array}$ & $\begin{array}{c}1.801 \\
515\end{array}$ & $\begin{array}{c}0.921 \\
501\end{array}$ & $\begin{array}{c}1.116 \\
738\end{array}$ & $\begin{array}{c}0.153 \\
94\end{array}$ & $\begin{array}{c}14.3 \\
1 \%\end{array}$ & $\begin{array}{c}0.317 \\
237\end{array}$ & $\begin{array}{c}0.284 \\
803\end{array}$ & $\begin{array}{c}0.313 \\
425\end{array}$ & $\begin{array}{c}0.274 \\
846\end{array}$ & $\begin{array}{c}0.003 \\
81\end{array}$ & $\begin{array}{c}1.20 \\
\%\end{array}$ \\
\hline 11 & $\begin{array}{c}1.085 \\
194\end{array}$ & $\begin{array}{c}2.013 \\
84\end{array}$ & $\begin{array}{c}0.922 \\
259\end{array}$ & $\begin{array}{c}1.202 \\
697\end{array}$ & $\begin{array}{c}- \\
0.162 \\
94\end{array}$ & $\begin{array}{c}- \\
15.0 \\
1 \%\end{array}$ & $\begin{array}{c}0.343 \\
639\end{array}$ & $\begin{array}{c}0.301 \\
031\end{array}$ & $\begin{array}{c}0.316 \\
225\end{array}$ & $\begin{array}{c}0.277 \\
756\end{array}$ & $\begin{array}{c}- \\
0.027 \\
41\end{array}$ & $\begin{array}{c}- \\
7.98 \\
\%\end{array}$ \\
\hline 12 & $\begin{array}{c}1.102 \\
469\end{array}$ & $\begin{array}{c}2.028 \\
577\end{array}$ & \begin{tabular}{|c|}
0.929 \\
189
\end{tabular} & $\begin{array}{c}1.317 \\
237\end{array}$ & $\begin{array}{c}0 . \\
0.173 \\
28 \\
\end{array}$ & $\begin{array}{l}15.7 \\
2 \% \\
\end{array}$ & $\begin{array}{c}0.356 \\
608\end{array}$ & $\begin{array}{c}0.307 \\
923\end{array}$ & $\begin{array}{c}0.324 \\
643\end{array}$ & $\begin{array}{c}0.282 \\
535\end{array}$ & $\begin{array}{c}0.031 \\
97\end{array}$ & $\begin{array}{c}8.96 \\
\%\end{array}$ \\
\hline 13 & $\begin{array}{c}1.204 \\
151\end{array}$ & $\begin{array}{c}2.073 \\
018\end{array}$ & $\begin{array}{c}0.930 \\
631\end{array}$ & $\begin{array}{c}1.430 \\
201\end{array}$ & $\begin{array}{c}- \\
0.273 \\
52\end{array}$ & $\begin{array}{c}- \\
22.7 \\
1 \%\end{array}$ & $\begin{array}{c}0.380 \\
635\end{array}$ & $\begin{array}{c}0.314 \\
827\end{array}$ & $\begin{array}{c}0.333 \\
335\end{array}$ & $\begin{array}{c}0.285 \\
584\end{array}$ & $\begin{array}{c}0.047 \\
3 \\
\end{array}$ & $\begin{array}{c}- \\
12.4 \\
3 \%\end{array}$ \\
\hline 14 & $\begin{array}{c}1.188 \\
8\end{array}$ & $\begin{array}{c}1.886 \\
015\end{array}$ & $\begin{array}{c}0.933 \\
138\end{array}$ & $\begin{array}{c}1.521 \\
9\end{array}$ & $\begin{array}{c}- \\
0.255 \\
66\end{array}$ & $\begin{array}{c}- \\
21.5 \\
1 \%\end{array}$ & $\begin{array}{c}0.371 \\
231\end{array}$ & $\begin{array}{c}0.313 \\
168\end{array}$ & $\begin{array}{c}0.343 \\
927\end{array}$ & $\begin{array}{c}0.289 \\
668\end{array}$ & $\begin{array}{c}0.027 \\
3\end{array}$ & $\begin{array}{c}- \\
7.35 \\
\% \\
\end{array}$ \\
\hline 15 & $\begin{array}{c}1.174 \\
322\end{array}$ & $\begin{array}{c}1.770 \\
835\end{array}$ & $\begin{array}{c}0.941 \\
801\end{array}$ & $\begin{array}{c}1.570 \\
472\end{array}$ & $\begin{array}{c}0 . \\
0.232 \\
52 \\
\end{array}$ & $\begin{array}{c}19.8 \\
0 \% \\
\end{array}$ & $\begin{array}{c}0.377 \\
227\end{array}$ & $\begin{array}{c}0.316 \\
201\end{array}$ & $\begin{array}{c}0.351 \\
93\end{array}$ & $\begin{array}{c}0.292 \\
833\end{array}$ & \begin{tabular}{|c}
0.025 \\
3 \\
\end{tabular} & $\begin{array}{c}6.71 \\
\% \\
\end{array}$ \\
\hline 16 & $\begin{array}{c}1.133 \\
324\end{array}$ & $\begin{array}{c}1.675 \\
68\end{array}$ & $\begin{array}{c}0.958 \\
364\end{array}$ & $\begin{array}{c}1.599 \\
966\end{array}$ & $\begin{array}{c}- \\
0.174 \\
96\end{array}$ & $\begin{array}{c}- \\
15.4 \\
4 \%\end{array}$ & $\begin{array}{c}0.379 \\
323\end{array}$ & $\begin{array}{c}0.315 \\
672\end{array}$ & $\begin{array}{c}0.361 \\
067\end{array}$ & $\begin{array}{c}0.296 \\
953\end{array}$ & $\begin{array}{c}- \\
0.018 \\
26\end{array}$ & $\begin{array}{c}- \\
4.81 \\
\%\end{array}$ \\
\hline 17 & $\begin{array}{c}1.159 \\
177\end{array}$ & $\begin{array}{c}1.792 \\
063\end{array}$ & $\begin{array}{c}0.972 \\
781\end{array}$ & $\begin{array}{c}1.647 \\
167\end{array}$ & $\begin{array}{c}0.186 \\
4 \\
\end{array}$ & $\begin{array}{l}16.0 \\
8 \% \\
\end{array}$ & $\begin{array}{c}0.399 \\
966\end{array}$ & $\begin{array}{c}0.327 \\
103\end{array}$ & $\begin{array}{c}0.370 \\
475\end{array}$ & $\begin{array}{c}0.299 \\
625\end{array}$ & \begin{tabular}{|c}
0.029 \\
49 \\
\end{tabular} & $\begin{array}{c}7.37 \\
\% \\
\end{array}$ \\
\hline 18 & $\begin{array}{c}1.182 \\
339\end{array}$ & $\begin{array}{c}1.877 \\
758\end{array}$ & $\begin{array}{c}0.986 \\
071\end{array}$ & $\begin{array}{c}1.689 \\
155\end{array}$ & $\begin{array}{c}- \\
0.196 \\
27\end{array}$ & $\begin{array}{c}- \\
16.6 \\
0 \%\end{array}$ & $\begin{array}{c}0.395 \\
601\end{array}$ & $\begin{array}{c}0.328 \\
712\end{array}$ & $\begin{array}{c}0.375 \\
694\end{array}$ & $\begin{array}{c}0.301 \\
887\end{array}$ & $\begin{array}{c}- \\
0.019 \\
91\end{array}$ & $\begin{array}{c}- \\
5.03 \\
\%\end{array}$ \\
\hline 19 & $\begin{array}{c}1.158 \\
908\end{array}$ & $\begin{array}{c}1.934 \\
969\end{array}$ & $\begin{array}{c}0.996 \\
834\end{array}$ & $\begin{array}{c}1.730 \\
566\end{array}$ & $\begin{array}{c}- \\
0.162 \\
07\end{array}$ & $\begin{array}{c}- \\
13.9 \\
9 \%\end{array}$ & $\begin{array}{c}0.373 \\
434\end{array}$ & $\begin{array}{c}0.329 \\
083\end{array}$ & $\begin{array}{c}0.380 \\
513\end{array}$ & $\begin{array}{c}0.305 \\
938\end{array}$ & $\begin{array}{c}0.007 \\
078\end{array}$ & $\begin{array}{c}1.90 \\
\%\end{array}$ \\
\hline 20 & $\begin{array}{c}1.258 \\
746\end{array}$ & $\begin{array}{c}2.464 \\
841\end{array}$ & $\begin{array}{c}0.999 \\
715\end{array}$ & $\begin{array}{c}1.775 \\
405\end{array}$ & $\begin{array}{c}- \\
0.259 \\
03\end{array}$ & $\begin{array}{c}- \\
20.5 \\
8 \%\end{array}$ & $\begin{array}{c}0.382 \\
013\end{array}$ & $\begin{array}{c}0.339 \\
925\end{array}$ & $\begin{array}{c}0.380 \\
087\end{array}$ & $\begin{array}{c}0.306 \\
541\end{array}$ & $\begin{array}{c}0.001 \\
93\end{array}$ & $\begin{array}{c}0.50 \\
\%\end{array}$ \\
\hline
\end{tabular}

Table 6 The Results of Second Pass Beta Coefficients

\begin{tabular}{|c|c|c|c|}
\hline Length Intervals & Pre Event & Post Event & Difference \\
\hline 5 & -0.4521 & -0.03294 & 0.419171 \\
\hline
\end{tabular}

\begin{tabular}{|c|c|c|c|}
\hline 5 & -0.45211 & -0.03294 & 0.419171 \\
\hline 10 & -0.56201 & -0.01525 & 0.546765 \\
\hline 15 & -0.6017 & -0.04281 & 0.55889 \\
\hline 20 & -0.63303 & -0.10709 & 0.52594 \\
\hline
\end{tabular}




\section{Conclusion}

This empirical study examines the abnormal performance of sample securities by using mean adjusted model, market adjusted model and market model. The paper investigated the information content in security prices on the release of quarterly earnings announcement by using event study and Cohen et al. (1983) methodology. The result of the number of positive and negative AARs and CAARs show that there are more numbers of positive values than negative values during the event window of 61 days. This result shows that market has positively reacted on the release of the September 2012 quarterly earnings announcement. These results are tested using the non-parametric tests. The randomness was tested in the behavior of AAR values using Runs test and found that the observed excess return series are not random during the event window of 61 days for mean adjusted model and market model. The sign statistics shows significant values for overall period for all models and for all the portfolios except for bad news of market adjusted portfolio. Therefore, it is concluded that there is a significant difference between the number of positive and negative AAR. The $t$ test results of the study show that AARs and CAARs values are significant for majority of the days in the event window of 61 days. Therefore, we reject the hypothesis that AAR and CAAR values are close to zero. The exception to this conclusion seems to be the bad news portfolio as their values are insignificant for the market adjusted model. The result from Cohen et al. (1983) methodology shows poor price adjustments process as the value of beta are decreased proportionately. The $\mathrm{R}^{2}$ values are also decreased proportionately during the post event period and this shows poor price efficiency. The BETA2 are negatively signed in the post event period. Based on overall results, we conclude that there is a scope for abnormal profits for the investors since the market fails to incorporate the new information in security prices. The above discussion clearly shows that the Indian stock market fails to perceive information content in security prices when they are publicly available as discussed by Fama (1965, 1970). The quarterly earnings information can generate significant abnormal profits to the trades in Indian stock market. Based on these results we conclude that the domestic and global investors can estimate their revenue growth during the earnings news by observing the market closely. This indicated that Indian stock market responded asymmetrically to good and bad news earnings announcements of September 2012 quarter. In spite of development in technology, asymmetry exists in dissemination of the information in Indian stock market. These results call for regulatory authorities to make policy changes for the proper dissemination of information. This study covered larger sample from Indian market and therefore, results can be generalised and will help the global and domestic investors for their investment decisions. This research contributes to the theory of EMH literature of emerging markets like India.

\section{References}

1. Ball, R. \& P. Brown, 'An Empirical Evaluation of Accounting Income Numbers', Journal of Accounting Research, 6(2), 1968, 159-178.

2. Ball, R. \& S. Kothari, 'Security Returns around Earnings Announcements', The Accounting Review, 66(4), 1991, 718-738. 
3. Barclay, M. \& R. Litzenberger, 'Announcement Effects of New Equity Issues and the Use of Intraday Price Data', Journal of Financial Economics, 21(1), 1988, 71- 99.

4. Basu S., 'The Information Content of Price-Earnings Ratios', Financial Management, 4 (2), 1975, 53-64.

5. Beaver, W., 'The information content of annual earnings announcements', Journal of Accounting Research, Supplement 6, (Supplement) 1968, 67-92.

6. Belgaumi M. S., 'Efficiency of the Indian Stock Market: An Empirical Study', Vikalapa, 20 (2), 1995, 43-47.

7. Bernard, V. \& J. Thomas, 'Evidence That Stock Prices Do Not Fully Reflect the Implications of Current Earnings for Future Earnings', Journal of Accounting and Economics, 13 (4), 1990, 305-340.

8. Bernard, V. \& J. Thomas, 'Post-earnings Announcement Drift: Delayed Price Response or Risk Premium?', Journal of Accounting Research, 27(Supplement), 1989, 1-36.

9. Brown, P., \& J. Kennelly, 'The Information Content of Quarterly Earnings: An Extension and Some Further Evidence', Journal of Business, 45(3), 1972, 403415.

10. Brown, S., \& J. Warner, 'Measuring security price performance', Journal of Financial Economics, 8, 1980, 205-258.

11. Brown, S., \& J. Warner, 'Using daily stock returns: The case of event studies', Journal of Financial Economics , 14(1), 1985, 3-31.

12. Butler, K. \& Malaikah, C., 'Efficiency and Inefficiency in Thinly Traded Stock Markets: Kuwait and Saudi Arabia', Journal of Banking and Finance, 16, 1992, 197-210.

13. Campbell, J. Lo, A. \& MacKinlay, A., 'The Econometrics of Financial Markets', Princeton University Press, Princeton, 1997.

14. Chaturvedi, O., 'Anomalies based on P/E ratios: Empirical evidence from the Indian stock market', ICFAI Journal of Applied Finance, 6, (3), 2000a, 1-13.

15. Chaturvedi, O., 'Empirical anomalies based on unexpected earnings: The Indian experience', ICFAI Journal of Applied Finance, 6 (1), 2000b, 52-64.

16. Chiat, H. \& Finn, F., 'Random walks on the stock exchange of Singapore', Accounting and Finance, 23, 1983, 81-87.

17. Cohen, K., G. Hawawini, S. Maier, R. Schwartz \& D. Whitcomb, 'Friction in the trading process and the estimation of systematic risk', Journal of Financial Economics, 1983, 264-278.

18. Cornell, B., \& W. Landsman, 'Security Price Response to Quarterly Earnings Announcements and Analysts' Forecast Revisions, The Accounting Review, 64(4), 1989, 680-692.

19. Cowles, A., 'Can stock market forecasters forecast?', Econometrica, 1(3), 1933, 309-324.

20. Dolley, J., 'Characteristics and procedure of common stock split-ups', Harvard Business Review, 11, 1933, 316-326.

21. Easton, P. \& Zmijewski, M, 'Cross sectional variation in the stock market response to accounting earnings announcement', Journal of Accounting and Economics, 12, 1989, 117-141.

22. Fama, E., 'Efficient Capital Markets: A Review of Theory and Empirical Work', Journal of Finance, 25(2), 1970, 383-417. 
23. Fama, E., 'Efficient Capital Markets: II', The Journal of Finance, 46(5), 1991, 1575-1617.

24. Fama, E., 'The behaviour of stock market prices', The Journal of Business, 11(1), 38, 1965, 34-105.

25. Fama, E., L. Fisher, M. Jensen \& R. Roll, 'The Adjustment of Stock Prices to New Information', International Economic Review, 10 (1), 1969, 1-21.

26. Foster, G., \& T. Shevlin, 'Earnings Releases, Anomalies, and the Behavior of Security Returns', The Accounting Review, 59 (4), 1984, 574-603.

27. Foster, G., 'Quarterly Accounting Data: Time-Series Properties and PredictiveAbility Results', The Accounting Review, 5 (2), 1977, 1-21.

28. Foster, T., \& D. Vickrey, 'The Information Content of Stock Dividend Announcements', Accounting Review, 53, (2), 1978, 360-370.

29. Frank, O. Evans, K. \& Newman, A. 'The Effect Of Earnings Announcement On Share Price Of Manufacturing Companies On The Ghana Stock Exchange', European Journal of Accounting, Auditing and Finance Research, 14(6), 2016, 96-111.

30. Grinblatt, M., R. Masulis, \& S. Titman, 'The valuation effects of stock splits and stock dividends,' Journal of Financial Economics', Harvard Business Review, 11, 1984, 316-326.

31. Gujarati, D. 'Basic Econometrics', New York, McGraw Hill Book Co, 2003.

32. Iqbal \& T. Mallikarjunappa, 'A Study of Efficiency of the Indian Stock Market', Indian Journal of Finance, 4 (5), 2010, 32-38.

33. Iqbal \& T. Mallikarjunappa, 'An Empirical Testing Of Semi-Strong Form Efficiency Of Indian Stock Market', The Journal of Amity Business School, 9, (1), 2008a, 24-33.

34. Iqbal \& T. Mallikarjunappa, 'Efficiency of stock market- A study of stock price response to earnings announcements', Published by Lambert Acad Publ. (2011)

35. Iqbal \& T. Mallikarjunappa, 'Quarterly Earnings Information, Stock Returns and Market Efficiency: An Empirical Study', An International B1-Annual Refereed Journal of Management and Technology, 2 (2), 2008b, 37-52.

36. Iqbal \& T. Mallikarjunappa, 'Stock price reactions to earnings announcement', ACRM Journal of Business and Management Research, 2(1), 2007, 10-15.

37. Iqbal, T. Mallikarjunappa \& N. Nayak 'Stock Price Adjustments to Quarterly Earnings Announcements: A Test of Semi-Strong form of Market Efficiency', An International B1-Annual Refereed Journal of Management \& Technology, 1, (2), 2007, 25-42.

38. Jansen, I. \& Nikiforov, A, 'Fear and greed: A returns-based trading strategy around earnings announcements', Journal of Portfolio Management, 42(4), 2016, 88-95.

39. Jegadeesh, N. \& J. Livnat, 'Post-earnings-announcement drift: The role of revenue surprises', Financial Analysts Journal 62 (2), 2006, 22-34.

40. Jennings, R., L. Starks, 'Information content and the speed of stock price adjustment', Journal of Accounting Research, 23 (2), 1985, 336-350.

41. Joy, O., R. Litzenberger, \& R. McEnally, 'The Adjustment Of Stock Prices To Announcements Of Unanticipated Changes In Quarterly Earnings', Journal of Accounting Research 15, 1977, 207-225.

42. Kormendi, R. \& R. Lipe, 'Earnings innovations, earnings persistence and stock returns', Journal of Business, 60 (3), (1987), 323-345. 
43. Lakonishok, J., \& T. Vermaelen, 'Tax-Induced Trading Around Ex-dividend Days', Journal of Financial Economics 16(3), 1986, 287-319.

44. Latane, H., \& C. Jones, 'Standardized unexpected earmngs 1971-1977', Journal of Finance, 34(3), 1979, 717-724.

45. Levene, H., 'On the power function of tests of randomness based on runs up and down', Annals of Mathematical Statistics, 23(1), (1952), 34-56.

46. Lonie, A., G. Abeyratna C, 'Power, Sinclair, 'The stock market reaction to dividend announcements: A UK study of complex market signals', Journal of Economic Studies, 23(1), 1996, 32 - 52.

47. Mallikarjunappa, T. \& J. Dsouza, 'A Study of Quarterly Earnings Announcement and Stock Price Reactions', The IUP Journal of Applied Finance, 20, (4), 2014, 94-106.

48. Mallikarjunappa, T. \& J. Dsouza, 'A study of semi-strong form of market efficiency of Indian stock market', Amity Global Business Review, 8, (2), 2013, 60-68.

49. Mallikarjunappa, T., 'How do the Indian stock prices react to quarterly earnings?', ICFAI Journal of Applied Finance, 10 (3), 2004, 37- 48.

50. Masulis, R., 'The effects of capital structure change on security prices: A study of exchange offers', Journal of Financial Economics, 8, 1980, 139-177.

51. Mendenhall, W., D. Wackerly, \& R. Scheaffer, '15: Nonparametric statistics Mathematical statistics with applications', (Fourth ed.), PWS-Kent, 1989, 674679.

52. Menike, M. \& Wang, 'Stock Market Reactions to the Release of Annual Financial Statements: Case of the Banking Industry in Sri Lanka, European Journal of Business and Management, 5(31) 2013, 75-86.

53. Nichols, D. \& Tsay, J. 'Security Price Reactions to Long-Range Executive Earnings Forecasts', Journal of Accounting Research, 17(1), 1979, 140-155.

54. Obaidullah, M., 'Stock price adjustment to half yearly earnings announcementsA test of market efficiently', Charted Accountant 38, 1990, 922-924.

55. Patell, J., M. Wolfson, 'The intraday speed of adjustment of stock prices to earnings and dividend announcements', Journal of Financial Economics 13(2), 1984, 223-252.

56. Raja, M., J. Sudhahar, \& M. Selvam, 'Testing the semi-strong form efficiency of Indian stock market with respect to information content of stock split announcement -A study in IT industry', International Research Journal of Finance and Economics, 25, 2009, 7-20.

57. Rao N., 'The adjustment of stock prices to corporate financial policy announcements', Finance India, 8 (4), 1994, 941-953.

58. Rendleman Jr., R. Jones \& C. Latane, 'Empirical anomalies based on unexpected earnings and the importance of risk adjustments', Journal of Financial Economics, 10, 1982, 269-287.

59. Saravanakumar, S. \& A. Mahadevan, 'An Empirical Study on the Announcement of Corporate Quarterly Results in India', Journal of Business Theory and Practice, 1(1) 2013, 119-131.

60. Schwartz, R., 'Reshaping the Equity Markets a Guide for the 1990s', Harper Business, 1991, 423-425. 
61. Seghal, S. \& K. Bijoy, 'Stock Price Reactions to Earnings Announcements: Evidence from India', Vision: The Journal of Business Perspective, 19 (1), 2015, 25-36.

62. Sharpe, W., 'Capital Asset Prices: A Theory of Market Equilibrium under Conditions of Risk', Journal of Finance, 19 (3), 1964, 425- 442.

63. Srinivasan, R., 'Security prices behaviour associated with right issue related events', ICFAI Journal of Applied Finance, 3 (1), 1997. 50-62.

64. Sulistiawan, D. Hartono, J. Tandelilin, E. \& Supriyadi, 'Earnings Announcements and Competing Information: The Indonesian Evidence', Journal of Indonesian Economy and Business: JIEB. 29(1), 2014, 93-94.

65. Truong, C. \& Corrado, C. 'Options trading volume and stock price response to earnings announcements', Review of Accounting Studies, 19(1), 2014, 161-209.

66. Watts, R., 'Systematic 'abnormal' returns after quarterly earnings announcements', Journal of Financial Economics, 6 (2-3), 1978, 127-150.

67. William M., Cready \& G. Patricia 'The Information Content of Annual Reports: A Price and Trading Response Analysis', The Accounting Review. 66(2), 1991, 291-312.

68. Woodruff, C. \& A. Senchack, 'Intradaily Price-Volume Adjustments of NYSE Stocks to Unexpected Earnings', The Journal of Finance, 43 (2), 1988, 467-491.

69. Woolridge, J., 'Dividend changes and security prices', Journal of Finance 38 (5), 1983, 1607-1615.

\section{About Our Authors}

Janet Jyothi Dsouza is working as an Associate Professor in the Department of Management Studies, Ballari Institute of Technology and Management, Ballari. She has nine years of teaching experience. She is a Ph. D from Mangalore University. She is specialized in accounting and finance. She has presented research papers in national and international conferences. She has received four best research paper awards.

T Mallikarjunappa is a Chairman in the Department of Business Administration, Mangalore University, India. He has worked as dean of the faculty, finance officer, member of the Academic Council, Syndicate, etc. at Mangalore University. He has 32 years of postgraduate teaching and research experience. He is a $\mathrm{PhD}$ from Mysore University and Associate Member of ICAI, Calcutta. He is on the editorial board of AIMS International Journal of Management and other journals. $\mathrm{He}$ is also the reviewer of papers for journals. His papers are published in AIMS International Journal of Management, ICFAI Journal of Applied Finance, Decision, Vikalpa, Asian Academy of Management Journal of Accounting and Finance, etc. His specialization is accounting and finance. He was awarded the AIMS International Fellow, by AIMS International, Houston, at the 10th AIMS International conference held at Bangalore. He has other awards such as the best teacher in finance and outstanding management teacher and researcher. 